\title{
Controllability Analysis of a pair of 3D Dubins vehicles in formation
}

\author{
Hamal Marino $^{\mathrm{a}}$, Paolo Salaris ${ }^{\mathrm{b}}$, Lucia Pallottino ${ }^{\mathrm{a}}$ \\ ${ }^{a}$ Research Center "Enrico Piaggio", and the Dipartimento di Ingegneria dell'Informazione, \\ University of Pisa, Largo L. Lazzarino 2, 56100 Pisa, Italy. \\ e-mail: lucia.pallottino@unipi.it, hamal.marino@gmail.com \\ ${ }^{b}$ INRIA (Institut National de Recherche en Informatique et Automatique), Sophia Antipolis - \\ Méditerranée, Lagadic project team, 2004 route des Lucioles, 06902 Sophia Antipolis, France. \\ e-mail: paolo.salaris@inria.fr
}

\begin{abstract}
In this paper we consider the controllability problem for a system consisting of a pair of Dubins vehicles moving in a 3D space (i.e. pair of 3D-Dubins vehicles) while maintaining constant distance. Necessary and sufficient conditions for the existence of a limited control effort to steer the system between any two configurations are provided. The proposed controllability analysis and the developed motion planning algorithm are a step toward the solution of planning problems for example in case the robots are physically constrained to a payload to be deployed. Moreover, results obtained in this paper are relevant in order to solve formation control problems for multiple robots as aerial or underwater vehicles, which move in 3D spaces. Simulation results highlight the sufficiency of the obtained conditions showing that even from critical configurations an admissible control can be determined.
\end{abstract}

Keywords:

Controllability, vehicle formation, 3D planning, nonholonomic vehicles

\section{Introduction}

Motion planning algorithms have been actively studied in the literature and there are several methods based e.g. on visibility graphs, potential field techniques or randomized sampling (see [1] and references therein). Several challenges may arise including issues related to nonholonomic and dynamic constraints, modeling uncertainty, noisy models, partial sensory data, and real-time computation. 
Motion planning becomes particularly difficult and interesting (see e.g. [2]) when physical robots have to perform tasks in a truly $3 \mathrm{D}$ environment avoiding static or dynamic obstacles such as in disaster sites, underwater and aerial environments. In such scenario, multi-robot systems can perform tasks more efficiently than a single robot or can accomplish tasks not executable by a single one. Moreover, multi-robot systems have advantages, e.g. providing flexibility to the task execution by exploiting distributed sensing and actuation. Also in nature, several types of animals, such as insects, birds, or fishes, aggregate together, moving en masse or migrating in some directions, also known as swarm behavior. The term shoaling or schooling is used to refer specifically to swarm behavior in fishes which derives many benefits including also the increased hydrodynamic efficiency (cf. [3]).

The cooperation and coordination of multi-robot systems (i.e. formation control) has been object of considerable research efforts (see [4] for a detailed review and references therein). Formation control studies the problem of controlling multiple robots with different kinematics and sensory equipment so that they can maintain some given configuration constraints (e.g. distances) while moving as a whole group [5, 6, 7]. Many approaches of formation control have been proposed, such as behavior-based methods [8], leader-follower strategies [9], [10] and virtual structure approaches [11]. Various kinds of nonholonomic vehicles have been considered, such as ground vehicles (e.g. in [12]), aircraft (e.g. in [13]) and underwater vehicles (e.g. in [14]).

In order to solve challenging problems as motion planning algorithms and formation control as well as to plan optimal trajectories, it is important to analyze and prove the controllability of the system. A system is completely controllable if, for every pair of points $q_{1}$ and $q_{2}$ in the configuration space, there exists a control that steers the system from $q_{1}$ to $q_{2},[15,16]$.

Unlike other approaches, the analysis of a tight constraint on the distance to be maintained is herein considered. There are several application scenarios in which the motion of the robots can be physically constrained due to a load of large dimensions to be deployed. For example refer to [17], [18] or to [19] where a group of quadrotor rigidly attached to a payload is considered. Other examples of those type of applications can be found in the aerospace robotics such as the JPL's Robot Colonie project where two rovers must transport a large box [20] and in underwater cooperative manipulation systems [21].

Beside the applications, the problem has several interesting theoretical aspects among which the control input set depends on the system configurations and classical controllability results can not be directly applied. Moreover the high di- 
mensional system is controlled by constrained 3-dimensional controls, and the range of admissible controls depends on the configuration variables. The solution of such constrained problems is also crucial for the solution of optimal control problems in which a minimum safety distance must be guarantee during motion. Indeed, the optimal solution consists also of arcs along which the robot travel at constant distance, see e.g. [22].

In [23] the controllability of different pairs of identical nonholonomic vehicles (e.g. differential drive and car-like vehicles) moving in a plane while maintain a constant distance has been proved. Results obtained have then been used in order to prove the controllability and design a motion planning algorithm for formations of planar Dubins vehicles, [13, 24].

In this paper our purpose is to extend results of [13] to a system consisting of a pair of 3D-Dubins vehicles moving in a 3D space while maintaining constant distance. Extension to the $3 \mathrm{D}$ case is not straightforward due to a more complex vehicle model and thus maneuvers between configurations must be accordingly computed. This paper completes our previous conference paper [25] which furnishes only sufficient conditions for controllability. Here we provide a more restrictive condition that is proved to be both necessary and sufficient. Finally, a motion planning algorithm to drive the considered system between initial and final configuration that verify the necessary and sufficient condition.

The paper is organized as follows. In Section 2 the model of two three dimensional Dubins vechiles are presented with the inputs and distance constraints to be verified. In Section 3 the effects of the controls on the system are evaluated in order to simplify the controllability analysis performed next. In Section 4 necessary conditions for the system controllability are obtained in terms of system internal configurations. In Section 5 several basic movements and associated control laws are obtained. Such movements are then combined to steer the system between any two configurations as described in Section 6. The necessary conditions are thus proven to be also sufficient for controllability if verified by the initial and final configurations. Finally, simulations results to highlight the verification of the constraints and the system's behaviour under the proposed controls is reported in Section 7.

\section{Problem definition}

Consider a nonholonomic vehicle moving in a three dimensional space and let $\langle W\rangle=\left(O_{w}, X_{w}, Y_{w}, Z_{w}\right)$ be a fixed reference frame. In $\langle W\rangle$, the vehicle configuration is $\zeta(t)=(x(t), y(t), z(t), \varphi(t), \psi(t))$ where $\mathbf{q}=(x(t), y(t), z(t))$ is the 


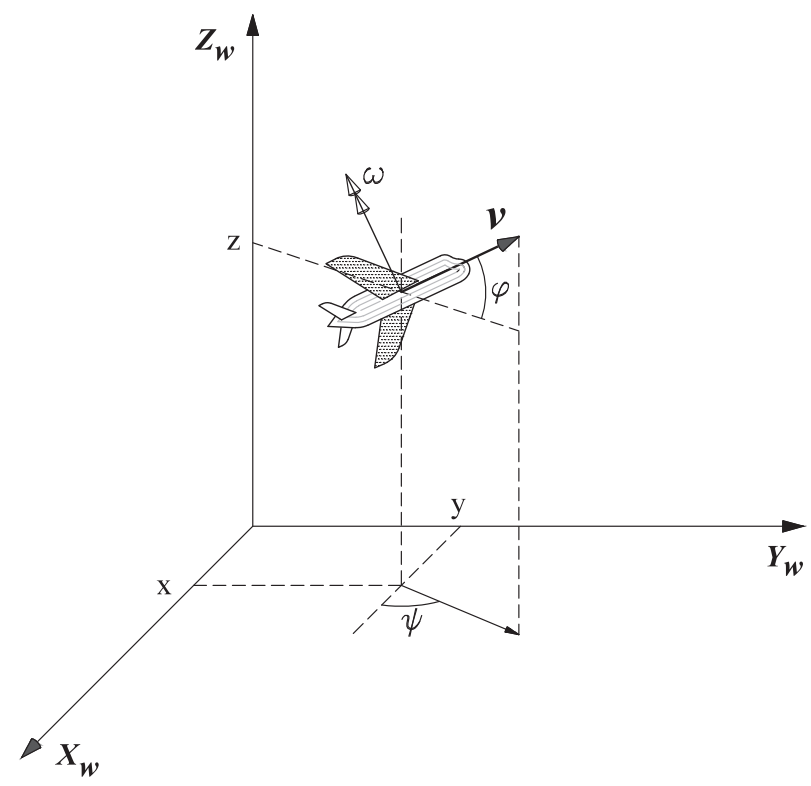

Figure 1: A single 3D-Dubins vehicle. The configuration $\zeta$ of the vehicle is described by three position variables $x, y$ and $z$ and two angular variable: $\varphi$ is the angle formed by the vehicle heading and the plane $X_{w} \times Y_{w}$ and $\psi$ is the angle formed by the projection of the vehicle heading on the plane $X_{w} \times Y_{w}$ and $X_{w}$ axis. The control inputs are the forward velocity $v=1$ and the angular velocity $\omega$.

position in $\langle W\rangle$ of the reference central point in the vehicle, $\varphi(t)$ is the angle formed by the vehicle heading and the plane $X_{w} \times Y_{w}$ and $\psi(t)$ is the angle formed by the projection of the vehicle heading on the plane $X_{w} \times Y_{w}$ and $X_{w}$ axis (see Fig. 1).

Given the forward velocity $v$ of the vehicle, the velocity vector $\mathbf{v}$ in $\langle W\rangle$ is $\mathbf{v}=$ $(v \cos \varphi \sin \psi, v \cos \varphi \cos \psi, v \sin \varphi)^{T}$. The kinematic model of the nonholonomic vehicle is (for the vehicle model for more details please refer to $[26,27]$ )

$$
\left\{\begin{array}{l}
\dot{\mathbf{q}}=\mathbf{v} \\
\dot{\mathbf{v}}=\mathbf{v} \times \boldsymbol{\omega}
\end{array}\right.
$$

where $\boldsymbol{\omega}=(\dot{\psi}, \dot{\varphi} \sin \psi,-\dot{\varphi} \cos \psi)^{T}$.

In this paper we consider the $3 D$-Dubins system that is described by system (1) subject to a constrained control effort $|\boldsymbol{\omega}| \leq \omega_{M}$. Moreover, without loss of generality, we consider $v=1$. In such conditions, a $3 D-D u b i n s$ generates trajectories with bounded curvature, as the minimum radius is $r=\frac{v}{\omega_{M}}$. 


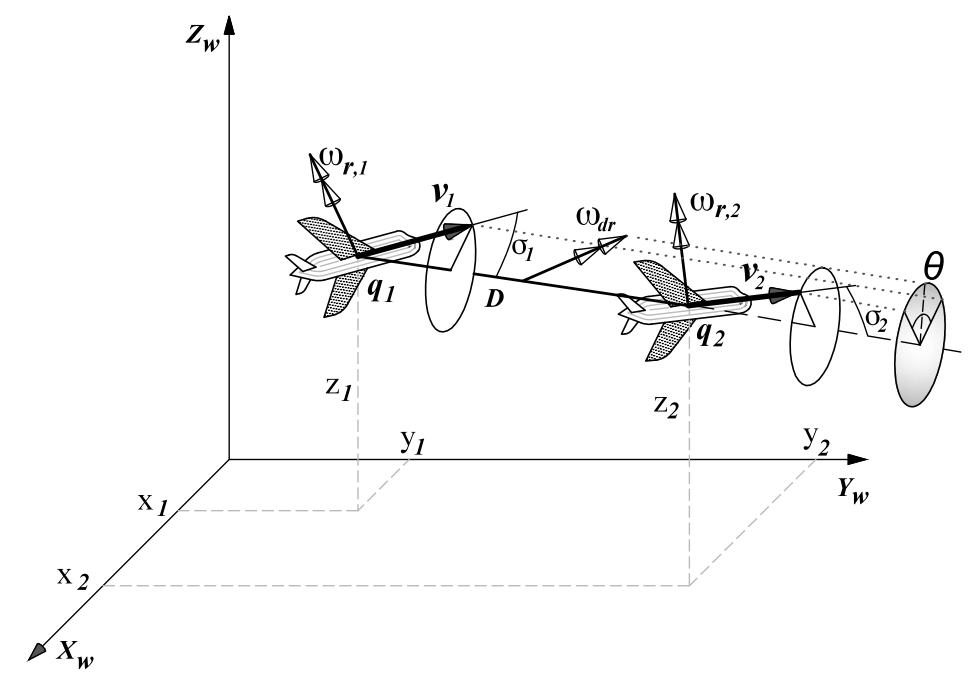

Figure 2: A pair of $3 D$-Dubins vehicles in formation. The relative configurations between the vehicles and/or the vector $\mathbf{D}$ are defined by variables $\sigma_{i}, i=1,2$ and $\theta$ where $\sigma_{i} \in[0, \pi / 2]$ is the angle between $\mathbf{v}_{i}$ and $\mathbf{D}(i=1,2)$ while $\theta \in[0, \pi]$ is the angle between the projections of $\mathbf{v}_{1}$ and $\mathbf{v}_{2}$ on the plane orthogonal to $\mathbf{D}$. The control effort $\boldsymbol{\omega}_{i}$ is decomposed in $\boldsymbol{\omega}_{d r}$ and $\boldsymbol{\omega}_{r, i}$ (see (12)).

Remark 1. For reader convenience we recall that the classical Dubins car is basically a unicycle vehicle moving on a plane with a constant positive forward velocity (usually normalized to 1) and a bounded angular velocity. The 3D-Dubins car here introduced is a generalization of the classical one to move in a $3 D$ space. For detailed discussions on the model, the constraints, the controllability properties and optimal control results please refer to [28, 29, 30] and references therein.

Consider now the system consisting of a pair of $3 D-D u b i n s$ constrained to maintain constant the magnitude $D$ of the distance vector $\mathbf{D}$ joining the centers of the two robots. The system is hence given by

$$
\left\{\begin{array}{l}
\dot{\mathbf{q}}_{1}=\mathbf{v}_{1} \\
\dot{\mathbf{q}}_{2}=\mathbf{v}_{2} \\
\dot{\mathbf{v}}_{1}=\mathbf{v}_{1} \times \boldsymbol{\omega}_{1} \\
\dot{\mathbf{v}}_{2}=\mathbf{v}_{2} \times \boldsymbol{\omega}_{2}
\end{array} .\right.
$$

subject to the constraint of a constant magnitude of vector $\mathbf{D}=\mathbf{q}_{2}-\mathbf{q}_{1}$ and limited control efforts

$$
\left|\boldsymbol{\omega}_{\boldsymbol{i}}\right| \leq \omega_{M}
$$


In Fig. 2 the position $\left(\mathbf{q}_{i}\right)$, forward $\left(\mathbf{v}_{i}\right)$ and angular $\left(\boldsymbol{\omega}_{i}\right)$ velocity vectors of vehicle $i, i=1,2$ are reported. Since the amplitude of vectors $\mathbf{v}_{i}$ is assumed constant, the velocity vectors are univocally determined by angles $\sigma_{i}$ and $\theta$ where $\sigma_{i} \in[0, \pi / 2]$ is the angle between $\mathbf{v}_{i}$ and $\mathbf{D}(i=1,2)$ while $\theta \in[-\pi, \pi]$ is the angle between the projections of $\mathbf{v}_{1}$ and $\mathbf{v}_{2}$ on the plane orthogonal to $\mathbf{D}$. Notice that, when $\sigma_{i}, i=1,2$, is zero the velocity vector $\mathbf{v}_{i}$ is aligned with $\mathbf{D}$ and $\theta$ is not defined. With an abuse of notation, in this case we define $\theta=0$.

The relations between variables $\sigma_{i}$ and $\theta$ and the system states $\mathbf{v}_{i}$ can be obtained through the projections $\mathbf{v}_{i \perp}$ of the velocities onto the plane orthogonal to D:

$$
\mathbf{v}_{i \perp}=\mathbf{v}_{i}-\frac{1}{D^{2}}\left(\mathbf{v}_{i}^{T} \mathbf{D}\right) \mathbf{D} \text { for } i=1,2 .
$$

Indeed, we have

$$
\left\{\begin{array}{l}
\sigma_{i}=\arccos \frac{\mathbf{v}_{i}^{T} \mathbf{D}}{D} \\
\theta= \begin{cases}\arccos \frac{\mathbf{v}_{1 \perp}^{T} \mathbf{v}_{2 \perp}}{\left\|\mathbf{v}_{1 \perp}\right\|\left\|\mathbf{v}_{2 \perp}\right\|}, & \left\|\mathbf{v}_{i \perp}\right\| \neq 0 \\
0, & \left\|\mathbf{v}_{i \perp}\right\|=0\end{cases}
\end{array},\right.
$$

From geometrical considerations, it can be seen that $\left\|\mathbf{v}_{i \perp}\right\|=\sin \sigma_{i}$, i.e. $\left\|\mathbf{v}_{i \perp}\right\| \neq 0$ if and only if $\sigma_{i} \neq 0$.

The goal of the paper is to solve the

Problem 1. Given initial and final configurations of system (2), determine necessary and sufficient conditions for the existence of control inputs that steer the system while guaranteeing the distance constraint.

\section{Controls and configurations constraints}

To solve Problem 1 we are interested in determining the conditions on the control inputs and the states of the system that satisfy the constraint on the magnitude of vector $\mathbf{D}$ during the system evolution. In other words, we want to determine the controls that keep constant the magnitude of vector $\mathbf{D}=\mathbf{q}_{2}-\mathbf{q}_{1}$ for any configuration of the vehicles. For this purpose we will decompose the control vectors $\boldsymbol{\omega}_{i}$ into components of which some will be constrained to maintain the distance while other will be determined to steer the system without violating the control effort constraint. 
Remark 2. In order to maintain constant the magnitude D of $\mathbf{D}$ it must necessarily hold the relation

$$
\sigma_{1}=\sigma_{2} \triangleq \sigma,
$$

Indeed, velocity vectors $\mathbf{v}_{1}$ and $\mathbf{v}_{2}$ must have equal components along the vector distance $\mathbf{D}$ to avoid variations of its length. Hence, it must hold $v_{1} \cos \sigma_{1}=$ $v_{2} \cos \sigma_{2}$. Thus, as $v_{1}=v_{2}=1$ and $\sigma_{i} \in[0, \pi / 2]$, it is necessary to have $\sigma_{1}=\sigma_{2}$. Hence, in the rest of the paper we will consider only configurations with $\sigma_{1}=$ $\sigma_{2}=\sigma$.

As a consequence, variables $\sigma$ and $\theta$ denote the relative configurations of the vehicles. Notice that, given $\mathbf{v}_{1}$, the internal variable $\sigma_{1}=\sigma$ can be computed through the first equation in (5). Once $\theta$ is known also the vector $\mathbf{v}_{2}$ can be obtained.

The variation of $\mathbf{D}, \dot{\mathbf{D}}=\mathbf{v}_{2}-\mathbf{v}_{1}$, must be orthogonal to $\mathbf{D}$ itself to guarantee a constant magnitude $D$, i.e. it holds

$$
\mathbf{D}^{T}\left(\mathbf{v}_{2}-\mathbf{v}_{1}\right)=0,
$$

The variation $\dot{\mathbf{D}}$ of $\mathbf{D}$ can be written in the form $\dot{\mathbf{D}}=\mathbf{D} \times \boldsymbol{\omega}_{\mathrm{dr}}$ where $\boldsymbol{\omega}_{\mathrm{dr}}$ is the angular velocity of $\mathbf{D}$ (see Fig. 2). Hence, $\boldsymbol{\omega}_{\mathrm{dr}}$ is characterized by the relation

$$
\mathbf{D} \times \boldsymbol{\omega}_{\mathrm{dr}}=\mathbf{v}_{2}-\mathbf{v}_{1} .
$$

It is worth noting that being $\boldsymbol{\omega}_{\mathrm{dr}}$ an angular velocity, it does not change the magnitude $D$ of $\mathbf{D}$. Moreover, the component of $\boldsymbol{\omega}_{\mathrm{dr}}$ along $\mathbf{D}$, represents a rigid rotation of the formation with respect to $\mathbf{D}$ itself. Hence, it does not affect the vehicles' relative configurations $\sigma$ and $\theta$. Such effects of the angular velocity $\omega_{\mathrm{dr}}$ will be used next to determine the elementary maneuvers that will be used to prove controllability. Indeed, it holds

Proposition 1. Applying controls $\boldsymbol{\omega}_{1}=\boldsymbol{\omega}_{2}=\boldsymbol{\omega}_{d r}, \sigma_{1}=\sigma_{2}=\sigma$ and $\theta$ are constant during system evolution. Indeed, the same rotational velocity is applied to $\mathbf{v}_{1}, \mathbf{v}_{2}$, and $\mathbf{D}$, making the system rotating as a whole, and keeping the internal configuration constant.

A formal proof can be found in Appendix 10.1. As a consequence of previous Proposition, we may assume

$$
\boldsymbol{\omega}_{\mathrm{dr}}^{T} \mathbf{D}=0 .
$$


Thus, considering (8) and (9), $\left(\mathbf{v}_{2}-\mathbf{v}_{1}\right) \times \mathbf{D}=\left(\mathbf{D} \times \boldsymbol{\omega}_{\mathrm{dr}}\right) \times \mathbf{D}=-\left(\mathbf{D}^{T} \boldsymbol{\omega}_{\mathrm{dr}}\right) \mathbf{D}+$ $D^{2} \boldsymbol{\omega}_{\mathrm{dr}}$ hence

$$
\boldsymbol{\omega}_{\mathrm{dr}}=\frac{\left(\mathbf{v}_{2}-\mathbf{v}_{1}\right) \times \mathbf{D}}{D^{2}} .
$$

In terms of $\theta$ and $\sigma$, from (8) and (10), it holds

$$
\omega_{\mathrm{dr}}=\frac{\left|\mathbf{v}_{2}-\mathbf{v}_{1}\right|}{D}=\frac{2 \sin \frac{\theta}{2} \sin \sigma}{D} .
$$

Moreover, from (8), the vector $\boldsymbol{\omega}_{\mathrm{dr}}$ is orthogonal to $\mathbf{v}_{2}-\mathbf{v}_{1}$, i.e.

$$
\boldsymbol{\omega}_{\mathrm{dr}}^{T} \mathbf{v}_{1}=\boldsymbol{\omega}_{\mathrm{dr}}^{T} \mathbf{v}_{2}
$$

As a consequence the projection of $\boldsymbol{\omega}_{\mathrm{dr}}$ and of $\mathbf{v}_{i}$ along the plane orthogonal to $\mathbf{D}$ form an angle $\theta / 2$, see Fig. 3 .

The control vectors $\boldsymbol{\omega}_{i}$ can be both written in terms of $\boldsymbol{\omega}_{\mathrm{dr}}$ as

$$
\boldsymbol{\omega}_{i}=\boldsymbol{\omega}_{\mathrm{dr}}+\boldsymbol{\omega}_{\mathrm{r}, i},
$$

where $\boldsymbol{\omega}_{\mathrm{r}, i}$ is the relative control that it is not necessarily orthogonal to $\boldsymbol{\omega}_{\mathrm{dr}}$. Moreover, $\boldsymbol{\omega}_{\mathrm{dr}}$ represents the component of the control that is common to both vehicles, it depends on the system configuration and hence it can not be chosen arbitrarily. In the following we will refer to $\boldsymbol{\omega}_{\mathrm{dr}}$ as the dragging control component. The assumption of orthogonality of the dragging control and the distance vector, in (9), is not restrictive since the parallel components of the angular velocity of $\mathbf{D}$ can be included in $\omega_{\mathrm{r}, i}$.

The distance constraint limits admissible configurations and controls that can be imposed to vehicles. Once the control is decomposed in the relative and dragging control components, the distance constraints does not depend on $\boldsymbol{\omega}_{\mathrm{dr}}$, indeed it holds

Proposition 2. Necessary conditions on system configurations and controls to guarantee the distance constraint (7) are $\sigma_{1}=\sigma_{2} \triangleq \sigma$, and

$$
\mathbf{D}^{T}\left(\mathbf{v}_{1} \times \boldsymbol{\omega}_{r, 1}\right)=\mathbf{D}^{T}\left(\mathbf{v}_{2} \times \boldsymbol{\omega}_{r, 2}\right) .
$$

Proof. Taking the time derivative of (7) and using (12) we have

$$
\begin{array}{r}
\left(\widehat{\omega}_{\mathrm{dr}} \mathbf{D}\right)^{T}\left(\mathbf{v}_{2}-\mathbf{v}_{1}\right)+\mathbf{D}^{T} \widehat{\omega}_{\mathrm{dr}}\left(\mathbf{v}_{2}-\mathbf{v}_{1}\right)+ \\
\mathbf{D}^{T}\left(\widehat{\omega}_{\mathrm{r}, 2} \mathbf{v}_{2}-\widehat{\omega}_{\mathrm{r}, 1} \mathbf{v}_{1}\right)
\end{array}=0 .
$$

where $\widehat{\omega}_{\mathrm{dr}}$ is the skew-symmetric matrix associated to the cross product, i.e. $\mathbf{v} \times$ $\boldsymbol{\omega}_{\mathrm{dr}}=\widehat{\omega}_{\mathrm{dr}} \mathbf{v}$. The first two addenda of (14) cancel out leading to $\mathbf{D}^{T} \widehat{\omega}_{\mathrm{r}, 2} \mathbf{v}_{2}=\mathbf{D}^{T} \widehat{\omega}_{\mathrm{r}, 1} \mathbf{v}_{1}$, hence the thesis. 
Remark 3. While the control component $\omega_{d r}$ can not be arbitrarily chosen, the component $\boldsymbol{\omega}_{r, i}$ is determined taking into account the constraints (3) and (13) to guarantee a limited control effort and a fixed distance between vehicles respectively. The choice of $\omega_{r, i}$ thus depends on the current configuration.

In order to increase the range of choices for input components $\boldsymbol{\omega}_{\mathrm{r}, i}$ it is possible to reduce the dragging control component as described next.

It is worth noting that a rotation of $\mathbf{v}_{i}$ along their axis, due to a non zero component of $\boldsymbol{\omega}_{i}$ along $\mathbf{v}_{i}$, does not influence the internal variables $\theta$ and $\sigma$ and hence it does not violate the distance constraint. Indeed, according to the considered model, all the rolling maneuvers of the vehicles does not modify the distance and hence can be neglected since the goal of this paper is to determine controls that steer the system among configurations without violating the distance constraint, i.e. a theoretical controllability result. However, such maneuvers can be exploited to compute and simplify the formation trajectories.

As a consequence, the component of the dragging control along $\mathbf{v}_{i}$ can be disregarded, while the orthogonal ones are

$$
\boldsymbol{\omega}_{\mathrm{dr}, i}^{\perp}=\boldsymbol{\omega}_{\mathrm{dr}}-\left(\boldsymbol{\omega}_{\mathrm{dr}}^{T} \mathbf{v}_{i}\right) \mathbf{v}_{i}, i=1,2,
$$

whose magnitudes are equal and, based on geometrical considerations, are

$$
\omega_{\mathrm{dr}}^{\perp}=\frac{\sin \left(\frac{\theta}{2}\right) \sin (\sigma) \sqrt{-2 \cos (\theta) \sin ^{2}(\sigma)+\cos (2 \sigma)+3}}{D} .
$$

In the particular case $\theta=\pi, \omega_{\mathrm{dr}}^{\perp}=\omega_{\mathrm{dr}}$.

From Remark 3, to have the maximum range of choice for the magnitude of the relative controls, it is convenient to choose also $\boldsymbol{\omega}_{\mathrm{r}, i}$ orthogonal to $\mathbf{v}_{i}$ for $i=1,2$ as follows. Let $\hat{\boldsymbol{u}}_{i}$ be the unit vectors directed as the dragging controls, i.e. $\boldsymbol{\omega}_{\mathrm{dr}, i}^{\perp}=\omega_{\mathrm{dr}}^{\perp} \hat{\boldsymbol{u}}_{i}$, and $\hat{\boldsymbol{u}}_{i}^{T} \mathbf{v}_{i}=0$, and let the unit vectors $\hat{\boldsymbol{w}}_{i}=\hat{\boldsymbol{u}}_{i} \times \mathbf{v}_{i}$, see Fig. 3 . Relative controls can hence be decomposed along $\hat{\boldsymbol{u}}_{i}$ and $\hat{\boldsymbol{w}}_{i}$ :

$$
\boldsymbol{\omega}_{\mathrm{r}, i}=u_{r, i} \hat{\boldsymbol{u}}_{i}+w_{r, i} \hat{\boldsymbol{w}}_{i} .
$$

Finally, the control on each vehicle $i=1,2$ will thus be written

$$
\boldsymbol{\omega}_{i}=\boldsymbol{\omega}_{\mathrm{dr}, i}^{\perp}+\boldsymbol{\omega}_{\mathrm{r}, i}=\left(\omega_{\mathrm{dr}}^{\perp}+u_{r, i}\right) \hat{\boldsymbol{u}}_{i}+w_{r, i} \hat{\boldsymbol{w}}_{i} .
$$

Notice that the directions $\hat{\boldsymbol{u}}_{i}, \hat{\boldsymbol{w}}_{i}$ are not well-defined when $\omega_{\mathrm{dr}}^{\perp}=0$ : nonetheless, in this case an arbitrary definition of these axes (still in the plane orthogonal to $\mathbf{v}_{i}$ ) will be suitable. 


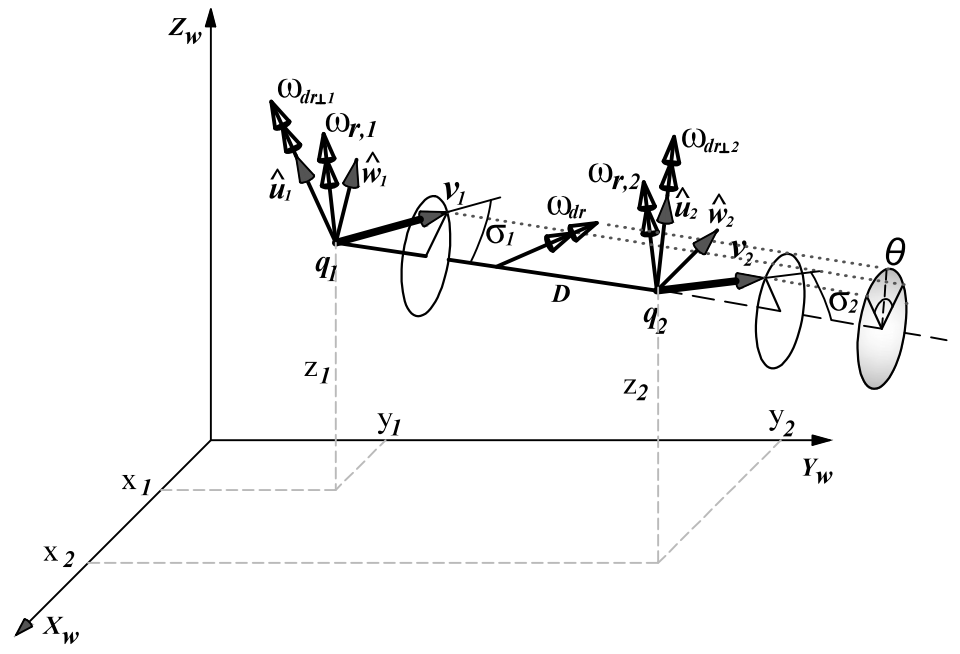

Figure 3: Decomposition of relative controls $\boldsymbol{\omega}_{\mathrm{r}, i}$ along directions $\hat{\boldsymbol{u}}_{i}$ and $\hat{\boldsymbol{w}}_{i}$ orthogonal to the velocities $\mathbf{v}_{i}$.

The last step is now to rewrite the necessary constraint (13) in terms of control components along $\hat{\boldsymbol{u}}_{i}$ and $\hat{\boldsymbol{w}}_{i}$. Substituting (16) in (13) and using properties of scalar and cross product we obtain (see Appendix 10.2.1-10.2.3)

$$
\begin{aligned}
& -\frac{u_{r, 1}}{\omega_{\mathrm{dr}}^{\perp}}\left(\mathbf{v}_{2}^{T} \mathbf{v}_{1}-1\right)+w_{r, 1} \mathbf{D}^{T} \hat{\boldsymbol{u}}_{1}= \\
& -\frac{u_{r, 2}}{\omega_{\mathrm{dr}}^{\perp}}\left(1-\mathbf{v}_{2}^{T} \mathbf{v}_{1}\right)+w_{r, 2} \mathbf{D}^{T} \hat{\boldsymbol{u}}_{2} .
\end{aligned}
$$

As shown in Appendix 10.2, $\mathbf{D}^{T} \hat{\boldsymbol{u}}_{1}=\mathbf{D}^{T} \hat{\boldsymbol{u}}_{2}$. Let

$$
\begin{gathered}
k_{1} \triangleq \frac{1-\mathbf{v}_{1}^{T} \mathbf{v}_{2}}{\omega_{\mathrm{dr}}^{\perp}}=\frac{2 \sin ^{2}\left(\frac{\theta}{2}\right) \sin ^{2} \sigma}{\omega_{\mathrm{dr}}^{\perp}} \\
k_{2} \triangleq \mathbf{D}^{T} \hat{u}_{i}=-\frac{\cos \sigma \sin \theta \sin ^{2} \sigma}{\omega_{\mathrm{dr}}^{\perp}}
\end{gathered}
$$

and also

$$
\alpha \triangleq \frac{k_{1}}{k_{2}}=-\frac{\tan \frac{\theta}{2}}{\cos \sigma} .
$$

Finally, necessary constraint can be rewritten as

$$
\alpha\left(u_{r, 1}+u_{r, 2}\right)+\left(w_{r, 1}-w_{r, 2}\right)=0
$$

that is linear in the control values $u_{r, i}$ and $w_{r, i}$. 


\section{Necessary condition for controllability}

The goal of this section is to determine conditions on internal system configurations that will be proven to be necessary for system controllability. We hence need first to determine those internal configurations for which controls that verify (3) exist. A possibility is to determine the minimum norm controls that verify the distance constraint. With this approach the desired control inputs are the solution of a convex optimization problem. Indeed, as the bound (3) applies to both vehicles, we can aim at minimizing the maximum between the norms of each control while keeping a constant distance. Hence, the goal is to solve the following MinMax problem:

Problem 2. Given $x=\left(u_{r, 1}, w_{r, 1}, u_{r, 2}, w_{r, 2}\right)$ and $\boldsymbol{\omega}_{i}=\left(\omega_{d r}^{\perp}+u_{r, i}\right) \hat{\boldsymbol{u}}_{i}+w_{r, i} \hat{\boldsymbol{w}}_{i}$, determine

$$
\left(\boldsymbol{\omega}^{*}\right)^{2}=\min _{x} f_{0}(x)=\min _{x}\left(\max \left(\left\|\boldsymbol{\omega}_{1}\right\|^{2},\left\|\boldsymbol{\omega}_{2}\right\|^{2}\right)\right)
$$

subject to

$$
h_{1}(x)=\alpha\left(u_{r, 1}+u_{r, 2}\right)+\left(w_{r, 1}-w_{r, 2}\right)=0 .
$$

Theorem 1. The controls that solve the convex optimization problem 2 are characterized by

$$
\begin{gathered}
u_{r, i}^{*}=-\frac{1}{1+\alpha^{2}} \omega_{d r}^{\perp} \\
w_{r, i}^{*}=(-1)^{i} \alpha u_{r, 1}^{*}=(-1)^{i+1} \frac{\alpha}{1+\alpha^{2}} \omega_{d r}^{\perp}
\end{gathered}
$$

and has norm

$$
\omega^{*}=\left\|\boldsymbol{\omega}_{i}^{*}\right\|=\sqrt{\frac{\alpha^{2}}{1+\alpha^{2}}} \omega_{d r}^{\perp}=2 \frac{\sin ^{2}\left(\frac{\theta}{2}\right) \sin \sigma}{D} .
$$

Proof. The optimization problem is convex since it consists of the minimization of a convex function $f_{0}(x)$ (i.e., a pointwise maximization of squared norms that are convex functions) and of a linear equality (hence convex) constraint $h_{1}(x)$. The cost function is not differentiable and a first order necessary condition of optimality in $x$ is given by

$$
0 \in \partial f_{0}(x)+\mu \partial h_{1}(x)
$$

where $\partial f(x)$ is the subdifferential of $f$ in $x$ and $\mu$ a multiplier (we refer to [31] and [32] for a detailed description of the theory of convex optimization problems). 
Since $h_{1}(x)$ is differentiable in $x$, its subdifferential is the gradient in $x$, i.e. $\partial h_{1}(x)=\left\{\nabla h_{1}(x)\right\}$ with $\nabla h_{1}(x)=(\alpha, 1, \alpha,-1)^{T}$. On the other hand the cost function $f_{0}(x)=\max \left(\left\|\boldsymbol{\omega}_{1}\right\|^{2},\left\|\boldsymbol{\omega}_{2}\right\|^{2}\right)$ is not differentiable in $\left\|\boldsymbol{\omega}_{1}\right\|=\left\|\boldsymbol{\omega}_{2}\right\|$ and the subdifferential in this case is given by the convex hull of the gradients of the two squared norms, i.e. for any subgradient $g \in \partial f_{0}(x)$ there exist non negative $\lambda_{1}$ and $\lambda_{2}$, with $\lambda_{1}+\lambda_{2}=1$, such that $g=\lambda_{1}\left(\omega_{\mathrm{dr}}^{\perp}+u_{r, 1}, w_{r, 1}, 0,0\right)^{T}+\lambda_{2}\left(0,0, \omega_{\mathrm{dr}}^{\perp}+\right.$ $\left.u_{r, 2}, w_{r, 2}\right)^{T}$. Otherwise, the subdifferential is the gradient of $\left\|\boldsymbol{\omega}_{1}\right\|^{2}\left(\left\|\boldsymbol{\omega}_{2}\right\|^{2}\right)$ if $f_{0}=\left\|\boldsymbol{\omega}_{1}\right\|^{2}>\left\|\boldsymbol{\omega}_{2}\right\|^{2}\left(f_{0}=\left\|\boldsymbol{\omega}_{2}\right\|^{2}>\left\|\boldsymbol{\omega}_{1}\right\|^{2}\right)$.

The necessary condition of optimality (23) requires the existence of a not null vector $\left(\lambda_{1}, \lambda_{2}, \mu\right)$ such that:

$$
\left(\begin{array}{ccc}
\omega_{\mathrm{dr}}^{\perp}+u_{r, 1} & 0 & \alpha \\
w_{r, 1} & 0 & 1 \\
0 & \omega_{\mathrm{dr}}^{\perp}+u_{r, 2} & \alpha \\
0 & w_{r, 2} & -1
\end{array}\right)\left(\begin{array}{c}
\lambda_{1} \\
\lambda_{2} \\
\mu
\end{array}\right)=\left(\begin{array}{l}
0 \\
0 \\
0 \\
0
\end{array}\right)
$$

Hence, imposing null determinants of submatrices, necessary conditions for optimality are:

$$
\left\{\begin{array}{l}
\omega_{\mathrm{dr}}^{\perp}+u_{r, 1}-\alpha w_{r, 1}=0 \\
\omega_{\mathrm{dr}}^{\perp}+u_{r, 2}+\alpha w_{r, 2}=0
\end{array}\right.
$$

By direct inspection of previous linear system both $\lambda_{1}$ and $\lambda_{2}$ must be non zero. Hence, the optimum is reached when $\left\|\boldsymbol{\omega}_{1}\right\|=\left\|\boldsymbol{\omega}_{2}\right\|$ (i.e. in the point of not differentiability of the cost function). Using (17) and (24), we have that the optimum is hence obtained when $w_{r, 1}= \pm w_{r, 2}$.

In case of $w_{r, 1}=w_{r, 2}$, the conditions (24) and the constraint (20) are verified only if $\omega_{\mathrm{dr}}^{\perp}=0$ and $u_{r, 1}=-u_{r, 2}=\alpha w_{r, 1}$. The value of the cost function is $u_{r, 1}^{2}+$ $w_{r, 1}^{2}=\left(1+\alpha^{2}\right) w_{r, 1}^{2}$. Hence, when $\omega_{\mathrm{dr}}^{\perp}=0$, the best choice is $w_{r, 1}=w_{r, 2}=u_{r, 1}=$ $u_{r, 2}=0$ that corresponds to a zero control.

In case of $w_{r, 1}=-w_{r, 2}$, the conditions (24) and the constraint (20) implies $u_{r, 1}=u_{r, 2}=-\frac{1}{1+\alpha^{2}} \omega_{\mathrm{dr}}^{\perp}$ and $w_{r, 1}=\frac{\alpha}{1+\alpha^{2}} \omega_{\mathrm{dr}}^{\perp}$. The value of the cost function is $\frac{\alpha^{2}}{1+\alpha^{2}}\left(\omega_{\mathrm{dr}}^{\perp}\right)^{2}$. Hence the thesis.

Remark 4. In the particular case of $\theta=\pi$, the optimal solution of the Problem 2 coincides with the dragging control, i.e. $\boldsymbol{\omega}_{1}^{*}=\boldsymbol{\omega}_{2}^{*}=\boldsymbol{\omega}_{d r}$. Indeed, for $\theta=\pi$ the constraint (20) reduces $h_{1}(x)=u_{r, 1}+u_{r, 2}=0$ while conditions (24) reduce to $w_{r, 1}=w_{r, 2}=0$. Hence, the minimum is attained for $u_{r, 1}=-u_{r, 2}=0$. Since, for $\boldsymbol{\theta}=\pi, \hat{\mathbf{u}}_{1}=\hat{\mathbf{u}}_{2}$ we have $\boldsymbol{\omega}_{1}^{*}=\boldsymbol{\omega}_{2}^{*}=\boldsymbol{\omega}_{d r}$. 
It is possible now to prove that the controls that solves Problem 2 do also guarantee that internal variables remain constant. Indeed, it holds

Proposition 3. For any pair of control vectors $\boldsymbol{\omega}_{i}$, optimal solutions of Problem 2 , i.e. such that the relative controls verify

$$
\begin{gathered}
w_{r, i}=(-1)^{i} \alpha u_{r, i}, \text { for } i=1,2, \\
u_{r, 1}=u_{r, 2},
\end{gathered}
$$

$\sigma$ and $\theta$ are constant during system evolution.

The proof can be found in Appendix 10.3 and, in particular, it proves also that the only condition (25a) of Proposition 3 is sufficient to ensure that the angle $\sigma$ is constant.

We are now able to prove

Theorem 2. The necessary condition for the existence of an admissible control, that steers the system among any two configuration, while respecting the distance constraint (7), is that

$$
2 \frac{\sin ^{2}\left(\frac{\theta}{2}\right) \sin \sigma}{D}<\omega_{M}
$$

at any time instant.

Proof. Consider an admissible control $\boldsymbol{\omega}$, i.e. with $\|\boldsymbol{\omega}\| \leq \omega_{M}$, that, by hypothesis, maintains a constant distance vector $\mathbf{D}$ between any given configuration pairs. Such control is a feasible variable of the optimization Problem 2 (it verifies the problem constraints) and hence it holds $\|\boldsymbol{\omega}\| \geq \omega^{*}$. Moreover, it necessarily holds that $\omega^{*} \leq \omega_{M}$, i.e. $2 \frac{\sin ^{2}\left(\frac{\theta}{2}\right) \sin \sigma}{D} \leq \omega_{M}$. We now want to prove the validity of the strict inequality. Consider initial and final configurations that strictly verify the condition (26). In case of equality, the control $\boldsymbol{\omega}$, with magnitude $\omega^{*}=\omega_{M}$, is a solution of the optimization problem and hence it verifies the hypothesis of Proposition 3. As a consequence, such control keeps constant the system internal variables and hence has constant maximum magnitude. Note that its magnitude can not be decreased since it has the minimum possible value. Hence, with such control, it is not possible to steer the system toward any final configuration for which a strict inequality holds. 


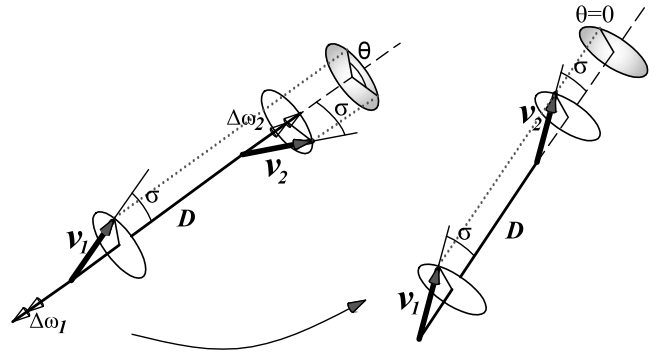

(a) Steering $\theta$ to zero (keeping constant $\sigma$ ) using $\boldsymbol{\omega}_{r, 1}$ and $\boldsymbol{\omega}_{r, 2}$ aligned along $\mathbf{D}$ and with opposite direction.

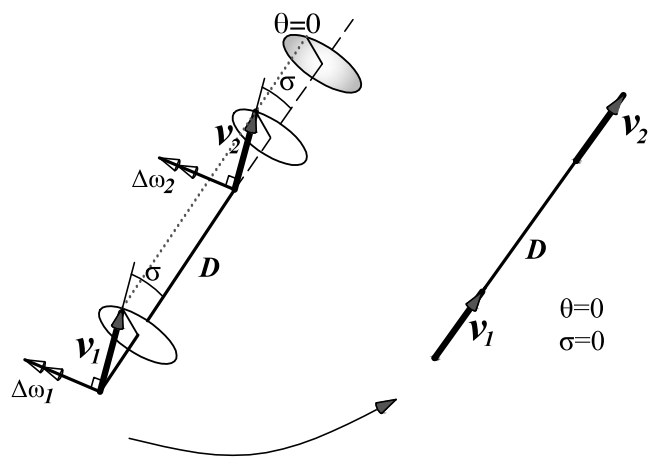

(b) Steering $\sigma$ to zero (keeping constant $\theta=0$ )

Figure 4: Steering $\theta$ and $\sigma$ to zero.

It is worth noting that, if $\omega_{M}>\frac{2}{D}$ the condition (26) is always verified. Moreover, in terms of minimum turning radius $R$ and desired distance value $D$, this occurs if $D>2 R$ that is the same condition to ensure complete controllability for a pair of planar Dubins vehicles, see [13]. In the rest of the paper we assume the more restrictive and interesting condition of $\omega_{M} \leq \frac{2}{D}$.

In the following, we will prove that the condition in (26) is also sufficient for the existence of controls that verify (3) while maintaining the desired distance, i.e. one of the main results of this paper. For this purpose we first introduce the concept of feasible configurations and then provide a motion planning algorithm to steer the system between feasible configurations while ensuring the control and distance constraints.

Definition 1. A configuration $\boldsymbol{\xi}=\left(\mathbf{q}_{1}, \mathbf{q}_{2}, \mathbf{v}_{1}, \mathbf{v}_{2}\right)$ will be called feasible if $\sigma_{1}=$ $\sigma_{2}=\sigma$ and $2 \frac{\sin ^{2}\left(\frac{\theta}{2}\right) \sin \sigma}{D}<\omega_{M}$, i.e. equations (6) and (26) hold.

Only feasible initial configurations are considered so that distance constraint is initially verified.

\section{Basic Movements}

In order to get a complete motion planning algorithm for a $3 D-D u b i n s$ pair, it is convenient to start investigating some basic movements and eventually connect them to form the complete path. Basic movements are defined based on the 
necessity to verify the control's admissibility condition, given by (3), along each movement. Given initial and final configurations, it is necessary to rotate and translate $\mathbf{D}$ accordingly. Based on results of previous sections we will now prove that there exist control inputs with limited amplitude that are able to regulate the rotation and translation of $\mathbf{D}$ independently among particular configurations characterized by $\sigma=\theta=0$. This lead to the choice of (basic) maneuvers to steer the system from generic configurations to such type of configurations (and viceversa) or among such particular configurations. Moreover, as it will be shown next, from such configurations translations and rotations of $\mathbf{D}$ will be obtained with planar movements. Hence, the three basic movements, described next, have been chosen in order to: a) steer $\sigma$ and $\theta$ to zero, b) purely rotate $\mathbf{D}$ and c) purely translate $\mathbf{D}$ (on a plane containing $\mathbf{D}$ ). The reverse of the first movement will be used to bring back $\sigma$ and $\theta$ to desired values.

From any feasible configuration, in order to have a margin of choice in the control, it is convenient to choose controls of the form $\boldsymbol{\omega}_{i}=\boldsymbol{\omega}_{i}^{*}+\boldsymbol{\Delta} \boldsymbol{\omega}_{i}$, where $\boldsymbol{\omega}_{i}^{*}$ are the controls associated to the solution of Problem 2 that depend on the internal configuration values. Recall that, from Proposition $3, \boldsymbol{\omega}_{i}^{*}$ keep the internal configurations $\theta$ and $\sigma$ constant. Hence, in the rest of the paper the value of $\boldsymbol{\Delta} \boldsymbol{\omega}_{i}$ will be determined to steer the system while guaranteeing $\left\|\omega_{i}\right\| \leq \omega_{M}$.

\subsection{Steering of $\theta$ and $\sigma$ to zero}

Steering $\theta$ to zero. Referring to Fig. $4(\mathrm{a})$, consider $\Delta \boldsymbol{\omega}_{1}$ and $\boldsymbol{\Delta} \boldsymbol{\omega}_{2}$ aligned along $\mathbf{D}$ so that the effect of relative controls on $\mathbf{D}$ is a rotation around its own axis and the distance constraint is not violated along system evolution.

Given an initial formation configuration $\boldsymbol{\xi}_{s}$, the function $S_{\theta}\left(\boldsymbol{\xi}_{s}\right)$ provides the configuration $\boldsymbol{\xi}_{1}$ reached by the formation subject to controls $\boldsymbol{\omega}_{i}$ with $\boldsymbol{\Delta} \boldsymbol{\omega}_{1}=-\boldsymbol{\Delta} \boldsymbol{\omega}_{2}$, parallel to $\mathbf{D}$ so that $\theta$ decreases and applied until $\theta$ is zero.

Steering $\sigma$ to zero. Once $\theta=0$ also $\boldsymbol{\omega}_{i}^{*}=0$ and hence $\boldsymbol{\omega}_{i}=\boldsymbol{\Delta} \boldsymbol{\omega}_{i} i=1,2$. Referring to Fig. 4(b), in order to align the two $3 D-$ Dubins on the distance vector, thus to rotate the vectors $\mathbf{v}_{i}$ towards $\mathbf{D}$ or equivalently to steer $\sigma$ to zero, it is sufficient to choose controls $\boldsymbol{\omega}_{i}$ orthogonal to $\mathbf{v}_{i}$ and $\mathbf{D}, i=1,2$.

Hence given an initial formation configuration $\xi_{1}$, with $\theta=0$, the function $S_{\sigma}\left(\boldsymbol{\xi}_{1}\right)$ provides the configuration ${ }^{a} \boldsymbol{\xi}_{s}$ reached by the formation while applying controls $\boldsymbol{\omega}_{i}$ with $\boldsymbol{\omega}_{1}=\boldsymbol{\omega}_{2}$, orthogonal to both $\mathbf{D}$ and $\mathbf{v}_{1}=\mathbf{v}_{2}$, until $\sigma$ is zero. Note that the equal magnitude is chosen to verify that $\sigma_{1}=\sigma_{2}=\sigma$ during evolution (see control constraint (13)). Moreover, in the configuration ${ }^{a} \boldsymbol{\xi}_{s}$ it holds $\theta=\sigma=0$. Hereafter, the superscript $a$ is used to denote that vehicles are aligned with $\mathbf{D}$. 
We refer to $S\left(\boldsymbol{\xi}_{s}\right)$ as the function that consisting in the composition of $S_{\theta}\left(\boldsymbol{\xi}_{s}\right)$ and $S_{\sigma}\left(\boldsymbol{\xi}_{1}\right)$ provides a configuration ${ }^{a} \boldsymbol{\xi}_{s}$ with $\theta=\sigma=0$.

\subsection{Steering of $\theta$ and $\sigma$ from zero}

With the function introduced in previous section, we are able to steer the system from any configuration to a configuration with $\sigma=\theta=0$. In order to perform the inverse maneuver the concept of reverse system is required. The reverse system of (2), is:

$$
\left\{\begin{array}{l}
\dot{\mathbf{q}}_{i}^{R}=-\mathbf{v}_{i}^{R} \\
\dot{\mathbf{v}}_{i}^{R}=-\mathbf{v}_{i}^{R} \times \boldsymbol{\omega}_{i}^{R} \quad, i=1,2 .
\end{array}\right.
$$

All symbols and subscripts are consistent to what we defined in (2). The only difference stands in the changed signs in the dynamics evolution.

In addition, the constraints represented by (3) and (7) also apply to this model with the appropriate variable substitution (e.g., $\mathbf{q}_{1}^{R}$ in place of $\mathbf{q}_{1}$ ).

Remark 5. Applying the control $\boldsymbol{\omega}(t)$ to system (2), from $\boldsymbol{\xi}\left(t_{0}\right)=\left(\mathbf{q}_{1}\left(t_{0}\right), \mathbf{q}_{2}\left(t_{0}\right)\right.$, $\left.\mathbf{v}_{1}\left(t_{0}\right), \mathbf{v}_{2}\left(t_{0}\right)\right)$ a configuration $\boldsymbol{\xi}\left(t_{f}\right)=\left(\mathbf{q}_{1}\left(t_{f}\right), \mathbf{q}_{2}\left(t_{f}\right), \mathbf{v}_{1}\left(t_{f}\right), \mathbf{v}_{2}\left(t_{f}\right)\right)$ is reached in $t=t_{f}$. In the reverse system, starting from $\boldsymbol{\xi}^{R}\left(t_{0}\right)=\boldsymbol{\xi}\left(t_{f}\right)$ and applying control $\boldsymbol{\omega}^{R}(t)=\boldsymbol{\omega}\left(t_{f}-t\right)$ (i.e. the reverse control law) the configuration $\boldsymbol{\xi}^{R}\left(t_{f}\right)=\boldsymbol{\xi}\left(t_{0}\right)$ is obtained.

As a consequence, given a configuration $\xi_{f}$, by applying the functions $S_{\theta}$ and $S_{\sigma}$ in the reverse systems (i.e. using the reverse controls) a configuration ${ }^{a} \boldsymbol{\xi}_{f}$ with $\sigma=\theta=0$ is obtained. We refer to $S^{R}\left(\boldsymbol{\xi}_{f}\right)$ as the function that given $\boldsymbol{\xi}_{f}$ returns ${ }^{a} \boldsymbol{\xi}_{f}$ such that by applying function $S(\boldsymbol{\xi})$ to $\boldsymbol{\xi}={ }^{a} \boldsymbol{\xi}_{f}$ the configuration $\boldsymbol{\xi}_{f}$ is reached.

\subsection{Rotation of $\mathbf{D}$ on a plane}

Consider now a configuration $a \boldsymbol{\xi}_{s}$ with $\theta=\sigma=0$ and a vector $\hat{\mathbf{D}}$ of length $D$. In order to align vector $\mathbf{D}$, in the initial configuration ${ }^{a} \boldsymbol{\xi}_{s}$, to $\hat{\mathbf{D}}$ we can perform a rotation around vector $\mathbf{D} \times \hat{\mathbf{D}}$ of angle $\delta=\arcsin \left(\frac{\|\mathbf{D} \times \hat{\mathbf{D}}\|}{D^{2}}\right)$.

When $\theta=0$, by rotating vectors $\mathbf{v}_{i}$ in opposite directions a rotation of $\mathbf{D}$ is induced and internal states are modified. The rotation occurs on the desired plane if controls $\Delta \boldsymbol{\omega}_{i}$ are parallel to $\mathbf{D} \times \hat{\mathbf{D}}$ but with opposite directions. The module of the control input must be equal to guarantee that the distance constraint is verified along the motion. Note that, applying such maneuver, $\theta=\pi$ as soon as $\sigma \neq 0$.

It is worth noting that by applying such non null inputs, $\Delta \boldsymbol{\omega}_{i}$, the angle $\sigma$ increases leading to a possible violation of the necessary condition (26). This may 
be avoided by choosing, if necessary, $\Delta \boldsymbol{\omega}_{i}=0$. Indeed, once $\sigma \neq 0$ the vector $\mathbf{D}$ rotates even though $\Delta \boldsymbol{\omega}_{i}=0$ due to the presence of $\omega_{i}^{*}$ (from Remark $4 \omega_{i}^{*}=\omega_{\mathrm{dr}}$ ) whose amplitude depends on the internal configurations values. Hence, in order to obtain a total rotation of amount $\delta$ a sequence of three controls can be used as described next.

Given a configuration ${ }^{a} \boldsymbol{\xi}_{s}$ with $\theta=\sigma=0$ and a vector $\hat{\mathbf{D}}$ of length $D$ consider the function $R\left({ }^{a} \boldsymbol{\xi}_{s}, \hat{\mathbf{D}}\right)$ that provides the configuration ${ }^{a} \boldsymbol{\xi}$ with $\theta=\sigma=0$ and $\mathbf{D}$ parallel to $\hat{\mathbf{D}}$. Consider a vector $\overline{\boldsymbol{\omega}}$ and the time instants $\bar{t}$ and $t_{1}$. The function is based on the composition of three controls:

1. $\Delta \boldsymbol{\omega}_{1}=-\Delta \boldsymbol{\omega}_{2}=\overline{\boldsymbol{\omega}}$ for $t \in\left[0, t_{1}\right)$,

2. $\Delta \boldsymbol{\omega}_{1}=\Delta \boldsymbol{\omega}_{2}=0$ (i.e. $\boldsymbol{\omega}_{i}=\boldsymbol{\omega}_{i}^{*}$ ) for $t \in\left[t_{1}, \bar{t}-t_{1}\right.$ ),

3. $\Delta \boldsymbol{\omega}_{1}=-\Delta \boldsymbol{\omega}_{2}=-\overline{\boldsymbol{\omega}}$ for $t \in\left[\bar{t}-t_{1}, \bar{t}\right]$.

With those choices of interval times and controls we have a symmetric behaviour of the system while controls 1 and 3 are applied. Hence, the amount of rotations performed by $\mathbf{D}$ is the same and if the necessary condition (26) is verified while applying control 1 it is also verified once control 3 is used.

It is now necessary to determine, based on the desired rotation amount $\delta$, the value of the three parameters in order to steer the formation while not violating the control input constraint (3). As abovementioned, the direction of vector $\overline{\boldsymbol{\omega}}$ must be parallel to $\mathbf{D} \times \hat{\mathbf{D}}$.

Consider a modulus $\overline{\boldsymbol{\omega}} \leq \omega_{M}$. While the constant magnitude control $\overline{\boldsymbol{\omega}}$ is applied it holds $\omega_{\mathrm{dr}}=\frac{2}{D} \sin (\bar{\omega} t)$, ref. to (11). Hence, the time needed to obtain a rotation of $\mathbf{D}$ of amplitude $\frac{\delta}{2}$ is $t_{\delta}$ such that $\frac{2}{D \bar{\omega}}\left(1-\cos \left(\bar{\omega} t_{\delta}\right)\right)=\delta / 2$. Note that the rest of the rotation will be performed while applying control 3 for the same amount of time.

On the other hand, the control input constraint must not be violated and it must hold $\omega_{\mathrm{dr}}+\bar{\omega} \leq \omega_{M}$. Indeed, from Remark 4, it holds that $\omega^{*}=\omega_{\mathrm{dr}}$. While applying control 1 , the time to reach the dragging control limit value $\omega_{\mathrm{dr}}=\omega_{M}-$ $\bar{\omega}$ is $t_{M}$ such that $\frac{2}{D} \sin \left(\bar{\omega} t_{M}\right)=\omega_{M}-\bar{\omega}$. Hence, the first control must be applied for time $t_{1}=\min \left\{t_{\delta}, t_{M}\right\}$.

If $\bar{\omega} \neq 0$ is such that $t_{1}=t_{\delta}<t_{M}$, the rotation of $\frac{\delta}{2}$ is obtained before that the control saturation occurs and hence before the necessary condition $\omega_{\mathrm{dr}} \leq \omega_{M}$ is violated. In this case, it is sufficient to choose the parameter $\bar{t}=2 t_{1}$ that corresponds to not apply control 2 while control 3 induces desired total rotation $\delta$ at time $2 t_{1}$. Moreover, it is worth noting that the symmetric control sequence steers the system toward a configuration that verifies $\theta=\sigma=0$. 
Otherwise, if $\bar{\omega}$ is such that $t_{1}=t_{M}<t_{\delta}$, a partial rotation of $\bar{\delta}=\frac{2}{D \bar{\omega}}(1-$ $\left.\cos \left(\bar{\omega} t_{M}\right)\right)$ is obtained (again the same amount of rotation is performed while applying control 3). At time $t_{M}$, the controls $\Delta \boldsymbol{\omega}_{i}=0$ can be applied. In other words the controls are $\boldsymbol{\omega}_{i}=\boldsymbol{\omega}_{i}^{*}=\boldsymbol{\omega}_{\mathrm{dr}}$ of magnitude $\omega_{M}-\overline{\boldsymbol{\omega}}$ and they keep $\theta=\pi$ and $\sigma=\bar{\omega} t_{M}$ constant (i.e. D rotates with constant angular velocity $\omega_{M}-\bar{\omega}$ ). Hence, time $\bar{t}$ is such that the remaining desired rotation is performed, i.e. it holds $\delta-2 \bar{\delta}=\left(\omega_{M}-\bar{\omega}\right)\left(\bar{t}-2 t_{M}\right)$. In this case the control sequence is effectively composed by three control values. Also in this case the sequence steers the system toward a configuration that verifies $\theta=\sigma=0$.

\subsection{Translation of $\mathbf{D}$ on a plane}

Given two formation configurations $a \xi$ and ${ }^{a} \boldsymbol{\xi}_{f}$ both with $\sigma=\theta=0$ and parallel distance vectors $\mathbf{D}$ the function $T\left({ }^{a} \xi,{ }^{a} \boldsymbol{\xi}_{f}\right)$ steers the formation between the two configurations with a planar translation.

Starting from a configuration with $\sigma=\theta=0$ consider the controls of equal amplitude and orthogonal to the plane containing the two configurations ${ }^{a} \xi$ and $a \boldsymbol{\xi}_{f}$. It is worth noting that, with this choice of controls, during the evolution $\theta \equiv 0$ and $\sigma_{1}=\sigma_{2}=\sigma$ and hence the formation is maintained.

In particular, it is possible to steer the system with circular arcs and straight lines choosing $\boldsymbol{\Delta} \boldsymbol{\omega}_{1}=\boldsymbol{\Delta} \boldsymbol{\omega}_{2}$ (i.e., $\boldsymbol{\omega}_{i}=\boldsymbol{\Delta} \boldsymbol{\omega}_{i}$ ) with magnitude smaller than $\omega_{M}$. It is worth noting that the necessary condition (26) is always verified since $\theta=0$. Hence, a parallel Dubins path can be computed between the two configurations consisting of parallel arcs of circles and straight lines, see e.g. [24], without rotations of $\mathbf{D}$, i.e. $\omega_{\mathrm{dr}}=0$.

\section{Necessary and Sufficient Condition for Controllability}

We are now interested in proving that the condition (26) in initial and final configurations is also sufficient to guarantee the existence of a control law that steers the system between the configurations without violating the distance and input constraints. In other words we are now able to prove the following

Theorem 3. Given internal configuration variables $\theta$ and $\sigma_{1}=\sigma_{2}=\sigma$ and the distance $D$ to be maintained, the condition

$$
2 \frac{\sin ^{2}\left(\frac{\theta}{2}\right) \sin \sigma}{D}<\omega_{M}
$$

at initial and final configurations, is both a necessary and sufficient condition for the controllability of the system. Where $\omega_{M}$ is the maximum control effort allowed. 


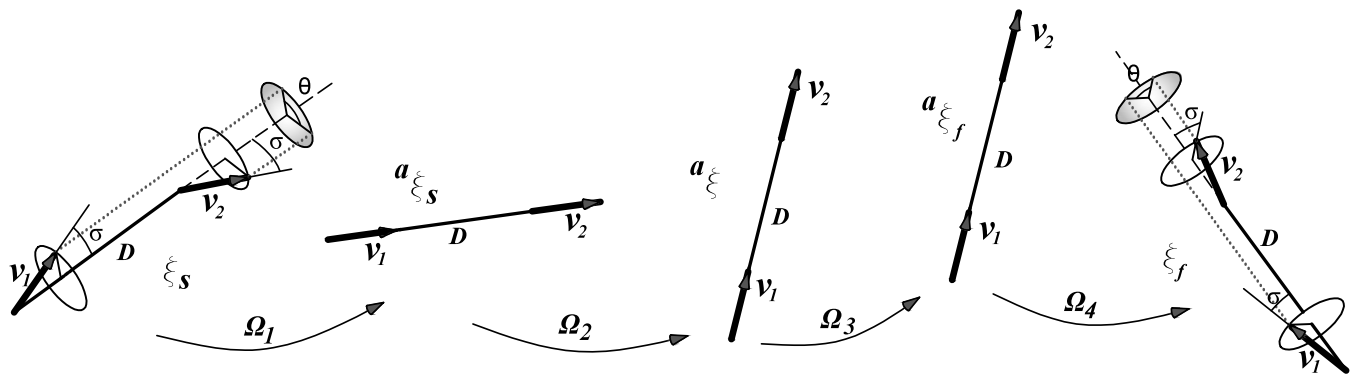

Figure 5: Motion planning steps. From the initial configuration $\boldsymbol{\xi}_{s}$, by applying the control $\boldsymbol{\Omega}_{1}$ a configuration ${ }^{a} \boldsymbol{\xi}_{s}$ such that $\theta=\sigma=0$ is reached and hence the vehicles are aligned with the vector $\boldsymbol{D}$. Starting from this configuration, by using the control $\boldsymbol{\Omega}_{2}$ the vector $\boldsymbol{D}$ rotates until the pair of vehicles reach a configuration ${ }^{a} \boldsymbol{\xi}$. In this new configuration the vector ${ }^{a} \boldsymbol{\xi}$ and ${ }^{a} \boldsymbol{\xi}_{f}$ differ by a simple translation. As a consequence, by applying control $\boldsymbol{\Omega}_{3}$ a parallel Dubins path is realized and the configuration ${ }^{a} \boldsymbol{\xi}_{f}$ is reached. Finally, by using the control $\boldsymbol{\Omega}_{4}=\boldsymbol{\Omega}_{1}^{R}$, the final configuration $\boldsymbol{\xi}_{f}$ is reached.

Proof. Theorem 2 states that the condition is necessary to ensure controllability of the system. Consider feasible initial and final configurations $\boldsymbol{\xi}_{s}$ and $\boldsymbol{\xi}_{f}$, i.e. configurations in which the condition (28) holds. We now need to prove that it exists a control sequence with limited modulus and that verifies the distance constraint during evolution between $\boldsymbol{\xi}_{s}$ and $\boldsymbol{\xi}_{f}$.

Denote with $\boldsymbol{\Omega}=\left(\boldsymbol{\omega}_{1}, \boldsymbol{\omega}_{2}\right)$ and as $\boldsymbol{\Omega}^{R}=\left(\boldsymbol{\omega}_{1}^{R}, \boldsymbol{\omega}_{2}^{R}\right)$ the controls of the original and reverse systems, respectively. Referring to Fig. 5, a motion planning algorithm can be obtained as follows:

1. Steer the system applying the control $\boldsymbol{\Omega}_{1}$, as described in section 5.1, until the configuration ${ }^{a} \boldsymbol{\xi}_{s}=S\left(\boldsymbol{\xi}_{s}\right)$ in which $\sigma=\theta=0$ is reached. In other words in ${ }^{a} \boldsymbol{\xi}_{s}$ vehicles are aligned to the distance vector.

2. Apply function $S^{R}(\boldsymbol{\xi})$ to $\boldsymbol{\xi}=\boldsymbol{\xi}_{f}$ to obtain the configuration ${ }^{a} \boldsymbol{\xi}_{f}=S^{R}\left(\boldsymbol{\xi}_{f}\right)$. Let $\hat{\mathbf{D}}$ be the distance vector of the formation in configuration ${ }^{a} \boldsymbol{\xi}_{f}$ and let ${ }^{a} \boldsymbol{\xi}=R\left({ }^{a} \boldsymbol{\xi}_{s}, \hat{\mathbf{D}}\right)$. Apply the control sequence $\boldsymbol{\Omega}_{2}$, described in section 5.3, to reach the configuration ${ }^{a} \boldsymbol{\xi}$ by rotating $\mathbf{D}$ until it is aligned with $\hat{\mathbf{D}}$;

3. Steer the system from ${ }^{a} \boldsymbol{\xi}$ to ${ }^{a} \boldsymbol{\xi}_{f}$ (with aligned vectors distance) through a parallel Dubins path applying control $\boldsymbol{\Omega}_{3}$ computed through $T\left({ }^{a} \boldsymbol{\xi},{ }^{a} \boldsymbol{\xi}_{f}\right)$ (ref. section 5.4);

4. Steer the system from ${ }^{a} \boldsymbol{\xi}_{f}$ to $\boldsymbol{\xi}_{f}$ applying controls $\boldsymbol{\Omega}_{4}$ obtained as the reverse of the controls $\boldsymbol{\Omega}_{1}^{R}$ used to steer the reverse system from $\boldsymbol{\xi}_{f}$ to ${ }^{a} \boldsymbol{\xi}_{f}$ with controls of type $\boldsymbol{\Omega}_{1}$. 


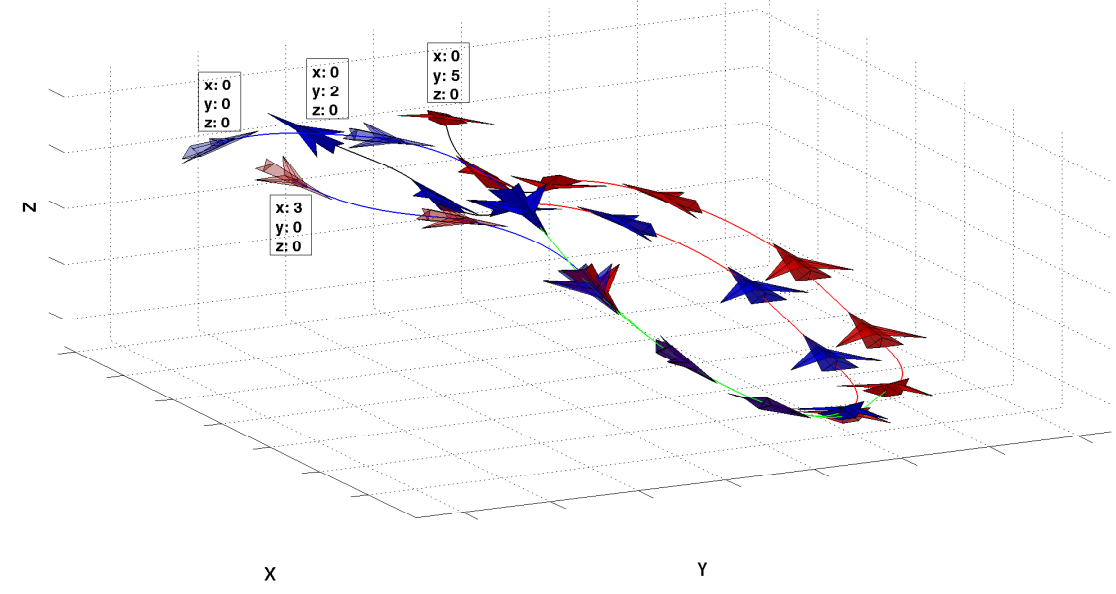

Figure 6: Path obtained from $\boldsymbol{\xi}_{s}=\left(0,0,0,3,0,0, \frac{1}{\sqrt{3}}, \frac{1}{\sqrt{3}}, \frac{1}{\sqrt{3}}, \frac{1}{\sqrt{3}}, \frac{1}{\sqrt{3}},-\frac{1}{\sqrt{3}}\right)^{T}$ to $\boldsymbol{\xi}_{f}=$ $\left(0,2,0,0,5,0,-\frac{1}{\sqrt{3}},-\frac{1}{\sqrt{3}}, \frac{1}{\sqrt{3}}, \frac{1}{\sqrt{3}},-\frac{1}{\sqrt{3}}, \frac{1}{\sqrt{3}}\right)^{T}$.

In summary, the steps are:

$$
\boldsymbol{\xi}_{s} \stackrel{\boldsymbol{\Omega}_{1}}{\longrightarrow} a \boldsymbol{\xi}_{s} \stackrel{\boldsymbol{\Omega}_{2}}{\longrightarrow} a \boldsymbol{\xi} \stackrel{\boldsymbol{\Omega}_{3}}{\longrightarrow} a \boldsymbol{\xi}_{f} \stackrel{\boldsymbol{\Omega}_{4}}{\longrightarrow} \boldsymbol{\xi}_{f}
$$

Note that, as mentioned in sections 5.1- 5.4 the controls in any step of the proposed algorithm maintain a constant distance between vehicles without violating the input constraint. Hence, the thesis.

\section{Simulations}

Consider initial and final configurations $\boldsymbol{\xi}_{s}^{T}=\left(\mathbf{q}_{1}\left(t_{0}\right)^{T}, \mathbf{q}_{2}\left(t_{0}\right)^{T}, \mathbf{v}_{1}\left(t_{0}\right)^{T}, \mathbf{v}_{2}\left(t_{0}\right)^{T}\right)$ and $\boldsymbol{\xi}_{f}^{T}=\left(\mathbf{q}_{1}\left(t_{f}\right)^{T}, \mathbf{q}_{2}\left(t_{f}\right)^{T}, \mathbf{v}_{1}\left(t_{f}\right)^{T}, \mathbf{v}_{2}\left(t_{f}\right)^{T}\right)$ where $\mathbf{q}_{1}\left(t_{0}\right)=(0,0,0)^{T}, \mathbf{q}_{2}\left(t_{0}\right)=$ $(3,0,0)^{T}, \mathbf{v}_{1}\left(t_{0}\right)=\left(\frac{1}{\sqrt{3}}, \frac{1}{\sqrt{3}}, \frac{1}{\sqrt{3}}\right)^{T}, \mathbf{v}_{2}\left(t_{0}\right)=\left(\frac{1}{\sqrt{3}}, \frac{1}{\sqrt{3}},-\frac{1}{\sqrt{3}}\right)^{T}, \mathbf{q}_{1}\left(t_{f}\right)=(0,2,0)^{T}$, $\mathbf{q}_{2}\left(t_{f}\right)=(0,5,0)^{T}, \mathbf{v}_{1}\left(t_{f}\right)=\left(-\frac{1}{\sqrt{3}},-\frac{1}{\sqrt{3}}, \frac{1}{\sqrt{3}}\right)^{T}$ and $\mathbf{v}_{2}\left(t_{f}\right)=\left(\frac{1}{\sqrt{3}},-\frac{1}{\sqrt{3}}, \frac{1}{\sqrt{3}}\right)^{T}$. The motion planning algorithm described in the proof of Theorem 3 will drive the system along the path represented in Fig. 6 maintaining a distance $D=3^{1}$.

\footnotetext{
${ }^{1}$ At https://youtu. be/El10SsKXy7o a video of the system evolution can be found.
} 


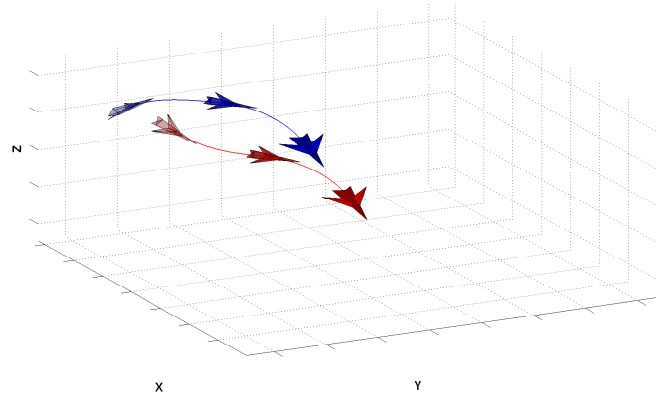

(a) Control $\boldsymbol{\Omega}_{\mathbf{1}}$

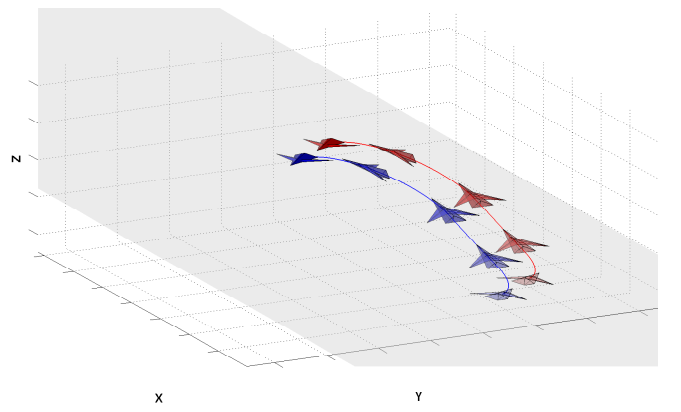

(c) Control $\boldsymbol{\Omega}_{\mathbf{3}}$

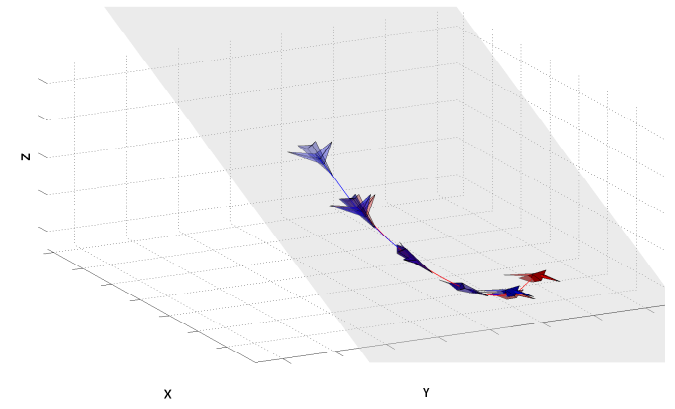

(b) Control $\boldsymbol{\Omega}_{\mathbf{2}}$

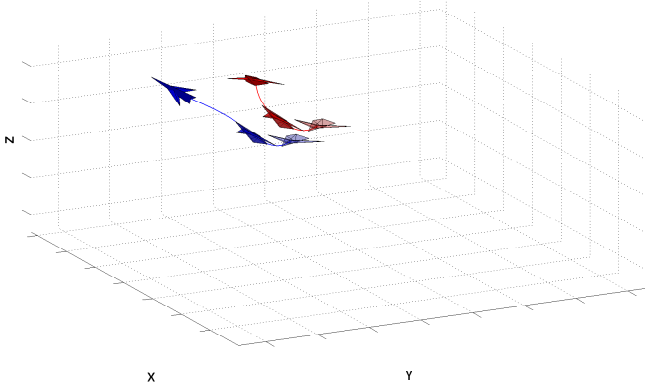

(d) Control $\mathbf{\Omega}_{\mathbf{4}}$

Figure 7: Steps of path represented in Fig. 6. Each picture represents the path that the vehicle follows by apply the control $\boldsymbol{\Omega}_{\boldsymbol{i}}$ as represented in Fig. 5 . Notice that, in the subfigures b) and c), the vehicles move on a plane.

To better highlight the planar evolution of the vehicles along parts of the path (in steps 2 and 3 of the algorithm), in Fig. 7 the step-by-step evolution for the path of Fig. 6 is represented. Moreover, in Fig. 8(a) the error with respect to the distance $D$ is reported to show the distance verification of the proposed control law which is in the order of $10^{-8}$ due to numerical errors. In Fig. 8(b) the evolution of $\sigma$ and $\theta$ is reported. Note that, according to basic movements described in 5, in the first and last steps $\sigma$ is constant while $\theta$ decreases from initial value to zero in step 1 and viceversa increases from zero to the final value in step 4. During step 2 the vector $\mathbf{D}$ is rotated while $\theta=\pi$. Finally in step 3 the vector $\mathbf{D}$ is translated while $\theta$ is zero.

In figures 9(a) and 9(b) the magnitudes of controls $\boldsymbol{\omega}_{1}, \boldsymbol{\omega}_{2}$ and $\boldsymbol{\omega}^{*}$ for the evolution of the system are reported. Notice that the magnitudes remain below the maximum allowed value $\omega_{M}$ chosen as $2 \frac{\sin ^{2}\left(\frac{\theta(0)}{2}\right) \sin \sigma(0)}{D}=0.314[\mathrm{rad} / \mathrm{s}]$. Furthermore, $\omega_{1}=\omega_{2}$ during steps 1,3 and 4 while when a rotation of $\mathbf{D}$ must be induced 


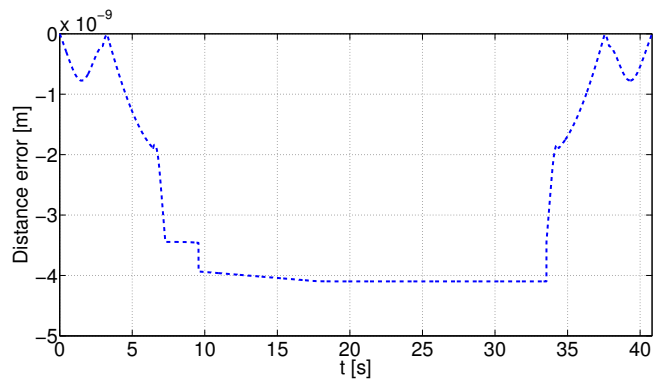

(a) Error on the required distance.

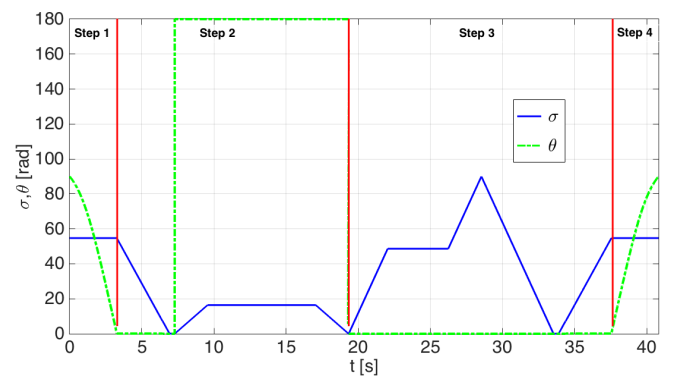

(b) Evolution of the internal variables $\sigma$ and $\theta$.

Figure 8: Evolutions of distance error and internal configurations values for path of Fig. 6. Notice that, in the plotting (a), the distance constraint is guaranteed along the whole path.

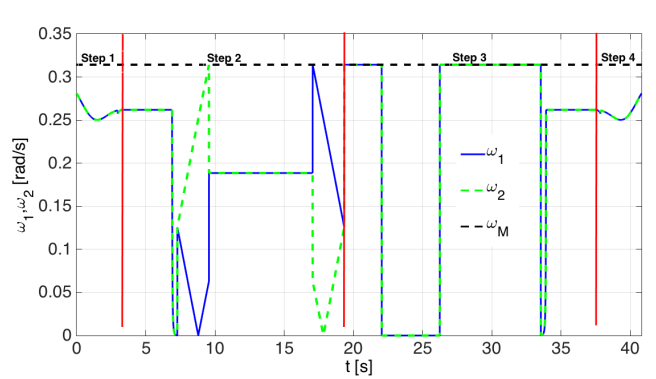

(a) Controls $\omega_{1}$ and $\omega_{2}$ and maximum allowed value $\omega_{M}$

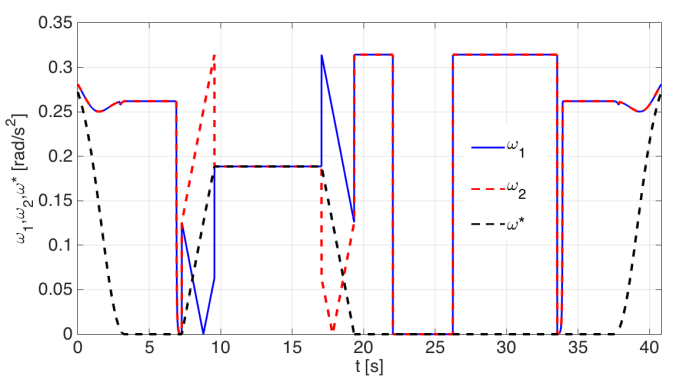

(b) Controls $\omega_{1}, \omega_{2}$ and $\omega^{*}$.

Figure 9: Evolutions of controls for path of Fig. 6. From the plotting (a) it is possible to verify that the maximum allowable effort control $\omega_{M}$ is always satisfied.

or stopped, they differ.

\section{Conclusions}

In this paper a model of a system consisting of a pair of Dubins vehicles moving in a 3D space maintaining constant distance has been considered with constraints on the controls. A motion planning algorithm of the proposed system has been introduced based on three basic movements with associated control laws. A theorem providing necessary and sufficient conditions on the initial and final configurations that guarantees the existence of admissible controls has been demonstrated based on the proposed motion planning algorithm. 
The results of this paper can be seen as a starting point to solve the motion planning and controllability problems for more complex formations of multivehicle systems as it has been done in $[13,24]$ for the planar case. Furthermore, the existence of a control that steers the system among feasible configurations is fundamental to solve the challenging optimal control problem, i.e. to determine the control law that steers the system as desired while minimizing a certain cost functional (e.g. time spent, distance travelled).

Future developments will also regard control laws that take into account more complex constraints depending on both states and controls, such as non uniform bounds on the control components and bounds on the roll angle.

\section{Acknowledgments}

The authors gratefully acknowledge H. Wang, M. Bonizzato and R. Bartalucci, for the useful discussions at the base of this work.

[1] S. M. LaValle, Planning Algorithms, Cambridge University Press, Cambridge, U.K., 2006, available at http://planning.cs.uiuc.edu/.

[2] J.-P. Laumond, D. Manocha, Editorial: Special issue on motion planning for physical robots, The International Journal of Robotics Research 32 (9-10) (2013) 989-990.

[3] M. Abrahams, P. Colgan, Fish schools and their hydrodynamic function: a reanalysis, Environmental Biology of Fishes 20 (1987) 79-80.

[4] Y. Chen, Z. Wang, Formation control: a review and a new consideration, in: Intelligent Robots and Systems (IROS). IEEE/RSJ International Conference on, 2005, pp. 3181-3186.

[5] F. Belkhouche, S. Vadhva, M. Vaziri, Modeling and controlling 3d formations and flocking behavior of unmanned air vehicles, in: Information Reuse and Integration (IRI), 2011 IEEE International Conference on, 2011, pp. 449 -454 .

[6] M. Mesbahi, M. Egerstedt, Graph theoretic methods in multiagent networks, Princeton University Press, 2010.

[7] L. Consolini, F. Morbidi, D. Prattichizzo, M. Tosques, A geometric characterization of leader-follower formation control, in: Robotics and Automation, 2007 IEEE International Conference on, IEEE, 2007, pp. 2397-2402. 
[8] T. Balch, R. Arkin, Behavior-based formation control for multirobot teams, IEEE Transactions on Robotics and Automation 14 (6) (1998) 926-939.

[9] A. Das, R. Fierro, V. Kumar, J. Ostrowski, J. Spletzer, C. Taylor, A visionbased formation control framework, IEEE Transactions on Robotics and Automation 18 (5) (2002) 813-825.

[10] J. Desai, J. Ostrowski, V. Kumar, Modeling and control of formations of nonholonomic mobile robots, IEEE Transactions on Robotics and Automation 17 (6) (2001) 905-908.

[11] M. Lewis, K. Tan, High precision formation control of mobile robots using virtual structures, Autonomous Robots 4 (4) (1997) 387-403.

[12] T. Barfoot, C. Clark, Motion planning for formations of mobile robots, Robotics and Autonomous Systems 46 (2) (2004) 65-78.

[13] H. Wang, L. Pallottino, A. Bicchi, Controllability properties for aircraft formations, in: Decision and Control (CDC), 2010 49th IEEE Conference on, 2010, pp. 2047 -2054. doi:10.1109/CDC.2010.5717824.

[14] R. Skjetne, S. Moi, T. I. Fossen, Nonlinear formation control of marine craft, in: Decision and Control, 2002, Proceedings of the 41st IEEE Conference on, Vol. 2, 2002, pp. 1699-1704 vol.2.

[15] J. P. Laumond, S. Sekhavat, Guidelines in nonholonomic motion planning for mobile robots, Robot Motion Planning and Control (1998) 1-53.

[16] H. Sussmann, G. Tang, Shortest paths for the Reeds-Shepp car: a worked out example of the use of geometric techniques in nonlinear optimal control, Technical Report SYNCON 91-10, Dept. of Mathematics, Rutgers University, Piscataway, NJ (1991).

[17] A. Yamashita, M. Fukuchi, J. Ota, T. Arai, H. Asama, Motion planning for cooperative transportation of a large object by multiple mobile robots in a $3 \mathrm{~d}$ environment, in: Robotics and Automation, 2000. Proceedings. ICRA '00. IEEE International Conference on, Vol. 4, 2000, pp. 3144-3151 vol.4.

[18] G. Gioioso, A. Franchi, G. Salvietti, S. Scheggi, D. Prattichizzo, The flying hand: A formation of uavs for cooperative aerial tele-manipulation, in: 2014 IEEE International Conference on Robotics and Automation (ICRA), 2014, pp. 4335-4341. 
[19] D. Mellinger, M. Shomin, N. Michael, V. Kumar, Cooperative grasping and transport using multiple quadrotors, in: Distributed autonomous robotic systems, Springer, 2013, pp. 545-558.

[20] W. Bluethmann, F. Rehnmark, T. Nguyen, H. Aldridge, Cooperative manipulation testbed: A facility for space robot control system development, in: International Symposium on Artificial Intelligence, Robotics and Automation in Space, 2001.

[21] N. Manerikar, G. Casalino, E. Simetti, S. Torelli, A. Sperind $\cong($, On cooperation between autonomous underwater floating manipulation systems, in: Underwater Technology (UT), 2015 IEEE, 2015, pp. 1-6.

[22] A. Bicchi, L. Pallottino, On optimal cooperative conflict resolution for air traffic management systems, IEEE Transactions On Intelligent Transportation Systems 1 (4) (2000) 221-231.

[23] H. Wang, L. Pallottino, A. Bicchi, Controllability for pairs of vehicles maintaining constant distance, IEEE International Conference on Robotics \& Automation, 2010 (2010) 342-349.

[24] H. Wang, L. Pallottino, A. Bicchi, Motion planning for formations of dubins vehicles, in: Decision and Control (CDC), 2010 49th IEEE Conference on, 2010, pp. 2263 -2269. doi:10.1109/CDC.2010.5717426.

[25] H. Marino, M. Bonizzato, R. Bartalucci, P. Salaris, L. Pallottino, Motion planning for two 3d-dubins vehicles with distance constraint, in: 2012 IEEE/RSJ International Conference on Intelligent Robots and Systems, 2012, pp. 4702-4707.

[26] H. J. Sussmann, Shortest 3-dimensional paths with a prescribed curvature bound, in: Decision and Control, 1995., Proceedings of the 34th IEEE Conference on, Vol. 4, 1995, pp. 3306-3312 vol.4.

[27] S. Hota, D. Ghose, Optimal geometrical path in 3d with curvature constraint, in: Intelligent Robots and Systems (IROS), 2010 IEEE/RSJ International Conference on, IEEE, 2010, pp. 113-118.

[28] J.-P. Laumond, Robot Motion Planning and Control, Springer-Verlag New York, Inc., Secaucus, NJ, USA, 1998. 
[29] L. Dubins, On curves of minimal length with a constraint on average curvature, and with prescribed initial and terminal positions and tangents, American Journal of Mathematics 79 (3) (1957) 497-516.

[30] T. Pecsvaradi, Optimal horizontal guidance law for aircraft in the terminal area, Automatic Control, IEEE Transactions on 17 (6) (1972) 763-772.

[31] N. Shor, K. Kiwiel, A. Ruszczynski, Minimization methods for nondifferentiable functions, Vol. 121, Springer-Verlag Berlin, 1985.

[32] S. Boyd, L. Vandenberghe, Convex optimization, Cambridge university press, 2004.

\section{Appendix}

\subsection{Proof of Proposition 1}

Proposition 2. Applying controls $\boldsymbol{\omega}_{1}=\boldsymbol{\omega}_{2}=\boldsymbol{\omega}_{d r}$ (i.e. considering $\boldsymbol{\omega}_{r, 1}=\boldsymbol{\omega}_{r, 2}=$ $0), \sigma_{1}=\sigma_{2}=\sigma$ and $\theta$ are constant during system evolution. Indeed, the same rotational velocity is applied to $\mathbf{v}_{1}, \mathbf{v}_{2}$, and to $\mathbf{D}$, making the system rotating as a whole, and keeping the internal configuration constant.

Proof. From the definition of $\sigma_{i}$ in (5) we have $\cos \sigma_{i}=\frac{1}{D} \mathbf{D}^{T} \mathbf{v}_{i} . \sigma_{1}=\sigma_{2}=\sigma$ and condition (13) holds for hypotheses. From Proposition 2, we have that $D$ is constant and hence it holds

$$
-\sin \sigma_{i} \dot{\sigma}_{i}=\frac{1}{D} \dot{\mathbf{D}}^{T} \mathbf{v}_{i}+\frac{1}{D} \mathbf{D}^{T} \dot{\mathbf{v}}_{i} .
$$

We recall that $\sin \sigma_{i}=\left\|\mathbf{v}_{i \perp}\right\|$ and $\dot{\mathbf{D}}=\mathbf{D} \times \boldsymbol{\omega}_{\mathrm{dr}}$. Furthermore, for hypothesis, $\dot{\mathbf{v}}_{i}=\mathbf{v}_{i} \times \boldsymbol{\omega}_{\mathrm{dr}}$, hence

$$
\dot{\sigma}_{i}=-\frac{1}{D\left\|\mathbf{v}_{i \perp}\right\|}\left(\left(\mathbf{D} \times \boldsymbol{\omega}_{\mathrm{dr}}\right)^{T} \mathbf{v}_{i}+\mathbf{D}^{T}\left(\mathbf{v}_{i} \times \boldsymbol{\omega}_{\mathrm{dr}}\right)\right)=0,
$$

where the last equality follows from

$$
\mathbf{a}^{T}(\mathbf{b} \times \mathbf{c})=-\mathbf{b}^{T}(\mathbf{a} \times \mathbf{c}) .
$$


From the definition of $\theta$ in (5) we have $\cos \theta=\frac{\mathbf{v}_{1 \perp}}{\left\|\mathbf{v}_{1 \perp}\right\|} \frac{\mathbf{v}_{2 \perp}}{\left\|\mathbf{v}_{2 \perp}\right\|}$. Notice that, under the hypothesis on the control variables, $\sigma_{i}$ are constant and hence $\left\|\mathbf{v}_{i \perp}\right\|=\sin \sigma_{i}$ are constant for $i=1,2$. Hence

$$
-\sin \theta \dot{\theta}=\frac{\dot{\mathbf{v}}_{1 \perp}}{\left\|\mathbf{v}_{1 \perp}\right\|} \frac{T}{\left\|\mathbf{v}_{2 \perp}\right\|}+\frac{\mathbf{v}_{1 \perp}}{\left\|\mathbf{v}_{1 \perp}\right\|} \frac{\dot{\mathbf{v}}_{2 \perp}}{\left\|\mathbf{v}_{2 \perp}\right\|} .
$$

To prove that $\theta$ is constant during evolution we need to prove that

$$
\dot{\mathbf{v}}_{1 \perp}^{T} \mathbf{v}_{2 \perp}+\mathbf{v}_{1 \perp}^{T} \dot{\mathbf{v}}_{2 \perp}=0 .
$$

From (4) we have

$$
\dot{\mathbf{v}}_{i \perp}=\dot{\mathbf{v}}_{i}-\frac{1}{D^{2}}\left(\left(\dot{\mathbf{v}}_{i}^{T} \mathbf{D}\right) \mathbf{D}+\left(\mathbf{v}_{i}^{T} \dot{\mathbf{D}}\right) \mathbf{D}+\left(\mathbf{v}_{i}^{T} \mathbf{D}\right) \dot{\mathbf{D}}\right) .
$$

Substituting $\dot{\mathbf{D}}=\mathbf{D} \times \boldsymbol{\omega}_{i}$ and $\dot{\mathbf{v}}_{i}=\mathbf{v}_{i} \times \boldsymbol{\omega}_{i}$ and using (30) we obtain that

$$
\dot{\mathbf{v}}_{i \perp}=\mathbf{v}_{i} \times \boldsymbol{\omega}_{i}-\frac{1}{D^{2}}\left(\mathbf{v}_{i}^{T} \mathbf{D}\right)\left(\mathbf{D} \times \boldsymbol{\omega}_{i}\right) .
$$

Notice that the last addendum in previous equation is orthogonal to $\mathbf{D}$ and hence computing $\dot{\mathbf{v}}_{i \perp}^{T} \mathbf{v}_{j \perp}$ the term related to $\frac{1}{D^{4}}$ is zero. Hence

$$
\begin{aligned}
& \dot{\mathbf{v}}_{i \perp}^{T} \mathbf{v}_{j \perp}=\left(\mathbf{v}_{i} \times \boldsymbol{\omega}_{i}\right)^{T} \mathbf{v}_{j}-\frac{1}{D^{2}}\left(\mathbf{v}_{j}^{T} \mathbf{D}\right)\left(\mathbf{v}_{i} \times \boldsymbol{\omega}_{i}\right)^{T} \mathbf{D} \\
& -\frac{1}{D^{2}}\left(\mathbf{v}_{i}^{T} \mathbf{D}\right)\left(\mathbf{D} \times \boldsymbol{\omega}_{i}\right)^{T} \mathbf{v}_{j}=\left(\mathbf{v}_{j} \times \mathbf{v}_{i}\right)^{T} \boldsymbol{\omega}_{i}+ \\
& \frac{1}{D^{2}}\left(\mathbf{D} \times \boldsymbol{\omega}_{i}\right)^{T}\left(\left(\mathbf{v}_{j}^{T} \mathbf{D}\right) \mathbf{v}_{i}-\left(\mathbf{v}_{i}^{T} \mathbf{D}\right) \mathbf{v}_{j}\right) .
\end{aligned}
$$

Notice that swapping $i$ with $j$ the equation changes in sign. Hence, under the hypothesis $\boldsymbol{\omega}_{1}=\boldsymbol{\omega}_{2}$ we have $\dot{\mathbf{v}}_{1 \perp}^{T} \mathbf{v}_{2 \perp}+\mathbf{v}_{1 \perp}^{T} \dot{\mathbf{v}}_{2 \perp}=0$.

\subsection{Useful results based on vector products identities}

In this section we report, for reader convenience, some computations based on scalar and cross products that are used in the paper.

\subsubsection{Computation of $\hat{\boldsymbol{u}}_{i}$}

From (15) we have $\hat{u}_{i}=\frac{\omega_{\mathrm{dr} \perp, i}}{\omega_{\mathrm{dr}}^{\perp}}$. Substituting (10) in (15), $\hat{u}_{i}$ can be obtained as function of $\mathbf{v}_{1}$ and $\mathbf{v}_{2}$ as follows and, thus, we obtain

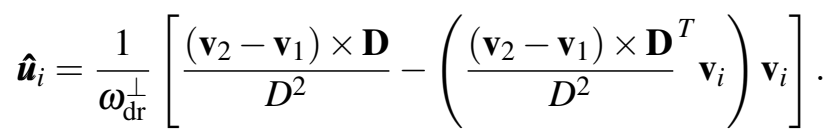


10.2.2. $\mathbf{v}_{i} \times\left(\hat{\boldsymbol{u}}_{i} \times \mathbf{v}_{i}\right)=\hat{\boldsymbol{u}}_{i}$

From the triple vector product it holds

$$
\mathbf{v}_{i} \times\left(\hat{\boldsymbol{u}}_{i} \times \mathbf{v}_{i}\right)=\left(\mathbf{v}_{i}^{T} \mathbf{v}_{i}\right) \hat{\boldsymbol{u}}_{i}-\left(\mathbf{v}_{i}^{T} \hat{\boldsymbol{u}}_{i}\right) \mathbf{v}_{i}=\hat{\boldsymbol{u}}_{i} .
$$

The last equation follows from the orthogonality of $\mathbf{v}_{i}$ and $\hat{\boldsymbol{u}}_{i}$ and the fact that $\mathbf{v}_{i}$ is a unit vector.

\subsubsection{Computation of $\mathbf{D}^{T}\left(\hat{\boldsymbol{u}}_{i} \times \mathbf{v}_{i}\right)$}

From the definition of $\hat{\boldsymbol{u}}_{i}$ and (33) we have

$$
\begin{aligned}
& \omega_{\mathrm{dr}}^{\perp} \hat{\boldsymbol{u}}_{i} \times \mathbf{v}_{i}= \\
& {\left[\frac{\left(\mathbf{v}_{2}-\mathbf{v}_{1}\right) \times \mathbf{D}}{D^{2}}-\left(\frac{\left(\mathbf{v}_{2}-\mathbf{v}_{1}\right) \times \mathbf{D}^{T}}{D^{2}} \mathbf{v}_{i}\right) \mathbf{v}_{i}\right] \times \mathbf{v}_{i}=} \\
& \frac{1}{D^{2}}\left[\left(\mathbf{v}_{2}-\mathbf{v}_{1}\right) \times \mathbf{D}\right] \times \mathbf{v}_{i}= \\
& \frac{1}{D^{2}}\left[\left(\mathbf{v}_{2}^{T} \mathbf{v}_{i}-\mathbf{v}_{1}^{T} \mathbf{v}_{i}\right) \mathbf{D}-\left(\mathbf{D}^{T} \mathbf{v}_{i}\right)\left(\mathbf{v}_{2}-\mathbf{v}_{1}\right)\right]
\end{aligned}
$$

where we have used the triple vector product in the last equation. Considering (7) we obtain

$$
\mathbf{D}^{T}\left(\hat{\boldsymbol{u}}_{i} \times \mathbf{v}_{i}\right)=\frac{1}{\omega_{\mathrm{dr}}^{\perp}}\left[\left(\mathbf{v}_{2}^{T} \mathbf{v}_{i}-\mathbf{v}_{1}^{T} \mathbf{v}_{i}\right)\right]
$$

\subsubsection{Computation of $\mathbf{v}_{1}^{T} \mathbf{v}_{2}$}

In order to compute a dot product, it is possible to choose an arbitrary reference frame. For simplicity, let us consider the reference frame where the $x$-axis is directed along $\mathbf{D}$, the $z$-axis is directed along $\boldsymbol{\omega}_{\mathrm{dr}}$, and the $y$-axis is chosen accordingly to the right-hand rule, i.e. $x \times y=z$.

In this frame, velocities form an angle $\sigma$ with the $x$-axis and have equal $z$ components. Components along the $y$-axis have same modulus and opposite sign. Finally, the angle between their projection on the $y-z$ plane and the $z$-axis is $\frac{\theta}{2}$. This translates into the following

$$
\begin{aligned}
& \mathbf{v}_{1}=\left[\begin{array}{lll}
\cos \sigma & \sin \sigma \sin \frac{\theta}{2} & \sin \sigma \cos \frac{\theta}{2}
\end{array}\right] \\
& \mathbf{v}_{2}=\left[\begin{array}{lll}
\cos \sigma & -\sin \sigma \sin \frac{\theta}{2} & \sin \sigma \cos \frac{\theta}{2}
\end{array}\right]
\end{aligned}
$$

The dot product is, thus

$$
\mathbf{v}_{1}^{T} \mathbf{v}_{2}=(\cos \sigma)^{2}+(\sin \sigma)^{2} \cos \theta .
$$

In the paper we will use also the identity

$$
\left(1-\mathbf{v}_{1}^{T} \mathbf{v}_{2}\right)=2\left(\sin \frac{\theta}{2}\right)^{2}(\sin \sigma)^{2}
$$




\subsubsection{Proof that $\mathbf{D}^{T} \hat{\boldsymbol{u}}_{1}=\mathbf{D}^{T} \hat{\boldsymbol{u}}_{2}$}

From the conditions $\mathbf{D}^{T} \boldsymbol{\omega}_{\mathrm{dr}}=0$ we obtain

$$
\begin{aligned}
& \mathbf{D}^{T} \hat{u}_{i}=\frac{1}{\omega_{\mathrm{dr}}^{\perp}} \mathbf{D}^{T} \boldsymbol{\omega}_{\mathrm{dr}, i}^{\perp}=\frac{1}{\omega_{\mathrm{dr}}^{\perp}} \mathbf{D}^{T}\left(\boldsymbol{\omega}_{\mathrm{dr}}-\left(\boldsymbol{\omega}_{\mathrm{dr}}^{T} \mathbf{v}_{i}\right) \mathbf{v}_{i}\right)= \\
& -\frac{1}{\omega_{\mathrm{dr}}^{\perp}}\left(\boldsymbol{\omega}_{\mathrm{dr}}^{T} \mathbf{v}_{i}\right)\left(\mathbf{D}^{T} \mathbf{v}_{i}\right)
\end{aligned}
$$

Since $\mathbf{D}^{T}\left(\mathbf{v}_{2}-\mathbf{v}_{1}\right)=\boldsymbol{\omega}_{\mathrm{dr}}^{T}\left(\mathbf{v}_{2}-\mathbf{v}_{1}\right)=0$, last term of (40) is independent on the value of $i=1$ or 2 . Hence the thesis.

Notice that, using the same reference frame defined in 10.2.4 and the value of $\omega_{\mathrm{dr}}$ in (11) we have

$$
\mathbf{D}^{T} \hat{\boldsymbol{u}}_{i}=-\frac{1}{\omega_{\mathrm{dr}}^{\perp}} \sin ^{2} \sigma \cos \sigma \sin \theta
$$

10.3. Proof of Proposition 3

Proposition 3. Given relative controls $\boldsymbol{\omega}_{r, i}$ verifying (20) and such that

$$
\begin{gathered}
w_{r, i}=(-1)^{i} \alpha u_{r, i}, \text { for } i=1,2, \\
u_{r, 1}=u_{r, 2},
\end{gathered}
$$

$\sigma_{1}, \sigma_{2}$ and $\theta$ are constant during system evolution.

Proof. Referring to the proof of Proposition 1 (in particular equation (29)) and considering $\dot{\mathbf{v}}_{i}=\mathbf{v}_{i} \times \boldsymbol{\omega}_{i}$, the condition

$$
\left(\mathbf{D} \times \boldsymbol{\omega}_{\mathrm{dr}}\right)^{T} \mathbf{v}_{i}+\mathbf{D}^{T}\left(\mathbf{v}_{i} \times \boldsymbol{\omega}_{i}\right)=0,
$$

implies $\dot{\sigma}_{i}=0$. Writing (43) in terms of relative controls $\boldsymbol{\omega}_{\mathrm{r}, i}$ and using (30), we have to prove that

$$
\mathbf{D}^{T}\left(\mathbf{v}_{i} \times \boldsymbol{\omega}_{\mathrm{r}, i}\right)=0 .
$$

Notice that, from the definition of $\hat{\boldsymbol{u}}_{i}$ and $\hat{\boldsymbol{w}}_{i}$ and the (42a), $\mathbf{D}^{T}\left(\mathbf{v}_{i} \times \boldsymbol{\omega}_{\mathrm{r}, i}\right)=\mathbf{D}^{T}\left(\mathbf{v}_{i} \times\right.$ $\left.\left(u_{r, i} \hat{\boldsymbol{u}}_{i}+w_{r, i} \hat{\boldsymbol{w}}_{i}\right)\right)=u_{r, i}\left(\mathbf{D}^{T}\left(\mathbf{v}_{i} \times \hat{\boldsymbol{u}}_{i}\right)-(-1)^{i} \alpha \mathbf{D}^{T} \hat{\boldsymbol{u}}_{i}\right)$. From (36), (39) and (41) we obtain $\mathbf{D}^{T}\left(\mathbf{v}_{i} \times \hat{\boldsymbol{u}}_{i}\right)-(-1)^{i} \alpha \mathbf{D}^{T} \hat{\boldsymbol{u}}_{i}=0$. Concluding, the angles $\sigma_{i}$ are constant under the only hypothesis (42a).

Regarding the angle $\theta$ the proof of Proposition 1 can be directly applied to the control under the hypotheses (3). Hence, since equation (31) is linear in $\boldsymbol{\omega}_{i}$, the common part $\boldsymbol{\omega}_{\mathrm{dr}}$ can be disregarded and it is sufficient to prove (31) in case $\boldsymbol{\omega}_{i}=\boldsymbol{\omega}_{\mathrm{r}, i}$. Equivalently, equation (32) holds with $\boldsymbol{\omega}_{i}=\boldsymbol{\omega}_{\mathrm{r}, i}$. Using the condition (44), equation (32) becomes

$$
\dot{\mathbf{v}}_{i \perp}^{T} \mathbf{v}_{j \perp}=\left(\mathbf{v}_{i} \times \boldsymbol{\omega}_{\mathrm{r}, i}\right)^{T} \mathbf{v}_{j}-\frac{1}{D^{2}}\left(\mathbf{v}_{i}^{T} \mathbf{D}\right)\left(\mathbf{D} \times \boldsymbol{\omega}_{\mathrm{r}, i}\right)^{T} \mathbf{v}_{j} .
$$


Given (7), $\mathbf{D}^{T} \mathbf{v}_{1}=\mathbf{D}^{T} \mathbf{v}_{2}$, it is sufficient to prove that $\left(\mathbf{v}_{1} \times \boldsymbol{\omega}_{\mathrm{r}, 1}\right)^{T} \mathbf{v}_{2}=-\left(\mathbf{v}_{2} \times\right.$ $\left.\boldsymbol{\omega}_{\mathrm{r}, 2}\right)^{T} \mathbf{v}_{1}$ and $\left(\mathbf{D} \times \boldsymbol{\omega}_{\mathrm{r}, 1}\right)^{T} \mathbf{v}_{2}=-\left(\mathbf{D} \times \boldsymbol{\omega}_{\mathrm{r}, 2}\right)^{T} \mathbf{v}_{1}$.

Consider first $\left(\mathbf{v}_{i} \times \boldsymbol{\omega}_{\mathrm{r}, i}\right)^{T} \mathbf{v}_{j}$. Notice that from the definition of $\hat{\boldsymbol{u}}_{i}$ we have $\left(\mathbf{v}_{1} \times \hat{\boldsymbol{u}}_{1}\right)^{T} \mathbf{v}_{2}=-\left(\mathbf{v}_{2} \times \hat{\boldsymbol{u}}_{2}\right)^{T} \mathbf{v}_{1}=\frac{1}{\omega_{\mathrm{dr}}^{\perp}}\left(\mathbf{v}_{2} \times \mathbf{v}_{1}\right)^{T} \boldsymbol{\omega}_{\mathrm{dr}}$. Moreover, from the condition $\boldsymbol{\omega}_{d r}^{T}\left(\mathbf{v}_{2}-\mathbf{v}_{2}\right)=0$ it holds $\mathbf{v}_{2}^{T} \boldsymbol{\omega}_{\mathrm{dr}}=\mathbf{v}_{1}^{T} \boldsymbol{\omega}_{\mathrm{dr}}$ and the definition of $\hat{\boldsymbol{u}}_{i}$ we have $\mathbf{v}_{1}^{T} \hat{\boldsymbol{u}}_{2}=$ $\mathbf{v}_{2}^{T} \hat{\boldsymbol{u}}_{1}=\frac{\mathbf{v}_{1}^{T} \boldsymbol{\omega}_{\mathrm{dr}}}{\omega_{\mathrm{dr}}^{\perp}}\left(1-\mathbf{v}_{1}^{T} \mathbf{v}_{2}\right)$. Hence, substituting $\boldsymbol{\omega}_{\mathrm{r}, i}=\omega_{i}^{*} \hat{\boldsymbol{u}}_{i}+(-1)^{i} \alpha \omega_{i}^{*}\left(\mathbf{v}_{i} \times \hat{\boldsymbol{u}}_{i}\right)$ in $\left(\mathbf{v}_{i} \times \boldsymbol{\omega}_{\mathrm{r}, i}\right)^{T} \mathbf{v}_{j}$, under hypothesis (42b), we obtain $\left(\mathbf{v}_{1} \times \boldsymbol{\omega}_{\mathrm{r}, 1}\right)^{T} \mathbf{v}_{2}=-\left(\mathbf{v}_{2} \times\right.$ $\left.\boldsymbol{\omega}_{\mathrm{r}, 2}\right)^{T} \mathbf{v}_{1}$.

Consider now $\left(\mathbf{D} \times \boldsymbol{\omega}_{\mathrm{r}, i}\right)^{T} \mathbf{v}_{j}$. From (44) and (10) we have $\left(\mathbf{D} \times \boldsymbol{\omega}_{\mathrm{r}, i}\right)^{T} \mathbf{v}_{j}=$ $\left(\mathbf{D} \times \boldsymbol{\omega}_{\mathrm{r}, i}\right)^{T}\left(\mathbf{v}_{j}-\mathbf{v}_{i}\right)=-\left(\mathbf{D} \times\left(\mathbf{v}_{j}-\mathbf{v}_{i}\right)\right)^{T} \boldsymbol{\omega}_{\mathrm{r}, i}=(-1)^{i+1} D^{2} \boldsymbol{\omega}_{\mathrm{r}, i}^{T} \boldsymbol{\omega}_{\mathrm{dr}}$. Finally, from (15), $\boldsymbol{\omega}_{\mathrm{r}, i}^{T} \boldsymbol{\omega}_{\mathrm{dr}}=\omega_{i}^{*} \omega_{t r \perp}$ hence the thesis. 